\title{
Modelling substorm chorus events in terms of dispersive azimuthal drift
}

\author{
A. B. Collier ${ }^{1,2}$ and A. R. W. Hughes ${ }^{1}$ \\ ${ }^{1}$ School of Pure and Applied Physics, University of KwaZulu-Natal, 4041, Durban, South Africa \\ ${ }^{2}$ Alfvén Laboratory, Royal Institute of Technology, SE-100 44, Stockholm, Sweden
}

Received: 4 March 2004 - Revised: 20 September 2004 - Accepted: 5 October 2004 - Published: 22 December 2004

\begin{abstract}
The Substorm Chorus Event (SCE) is a radio phenomenon observed on the ground after the onset of the substorm expansion phase. It consists of a band of VLF chorus with rising upper and lower cutoff frequencies. These emissions are thought to result from Doppler-shifted cyclotron resonance between whistler mode waves and energetic electrons which drift into a ground station's field of view from an injection site around midnight. The increasing frequency of the emission envelope has been attributed to the combined effects of energy dispersion due to gradient and curvature drifts, and the modification of resonance conditions and variation of the half-gyrofrequency cutoff resulting from the radial component of the $\boldsymbol{E} \times \boldsymbol{B}$ drift.

A model is presented which accounts for the observed features of the SCE in terms of the growth rate of whistler mode waves due to anisotropy in the electron distribution. This model provides an explanation for the increasing frequency of the SCE lower cutoff, as well as reproducing the general frequency-time signature of the event. In addition, the results place some restrictions on the injected particle source distribution which might lead to a SCE.
\end{abstract}

Key words. Space plasma physics (Wave-particle interaction) - Magnetospheric physics (Plasma waves and instabilities; Storms and substorms)

\section{Introduction}

The substorm chorus event is a recognised VLF signature of the substorm expansion phase (Smith et al., 1996, 1999). Although chorus emissions are commonly observed in the postmidnight sector in association with substorm activity (Tsurutani and Smith, 1974), the SCE is distinctive as it consists of a band of chorus with ascending upper and lower cutoff frequencies. The duration of a SCE is usually $\sim 10 \mathrm{~min}$ to $\sim 1 \mathrm{~h}$. The frequency of the leading edge of the emission envelope increases at a rate of between 20 and $1000 \mathrm{~Hz} / \mathrm{min}$ (Smith et al., 1996), but typically around $150 \mathrm{~Hz} / \mathrm{min}$ (Smith et al.,
2002). These events are commonly observed between midnight and dawn, following energetic particle injection into the nightside inner magnetosphere. The correlation of these two phenomena allows the substorm onset time to be estimated from the SCE epoch with an uncertainty of around 10 min (Smith et al., 1999).

Observations of SCE have been documented at a number of high-latitude stations including Eights $[L=3.8]$ (Carpenter et al., 1971), Roberval [ $L=4.0]$ (Carpenter et al., 1975), SANAE-III $[L=4.1]$ (Hughes, 1995; Collier and Hughes, 2004), Siple [ $L=4.2]$ (Carpenter et al., 1975; Park et al., 1981), Halley [ $L=4.4]$ (Smith et al., 1996; 1999; 2002), and Byrd [ $L=7.1]$ (Carpenter et al., 1971). Events have also been recorded simultaneously at conjugate stations (Carpenter et al., 1975) and observed by satellites near geosynchronous orbit (Isenberg et al., 1982). Observations made at SANAE-IV $[L=4.3]$ are presented in Sect. 2.

Smith et al. (1996) observed 243 SCE in 327 days at Halley during 1992, noting that the frequency of occurrence, around $250 \mathrm{y}^{-1}$ on average (Smith et al., 1999), was greater at geomagnetically disturbed $\left(K_{p}>3-\right)$ than quiet times. Substorm chorus events appear to be observed less often at SANAE-IV (fewer than 50 during 2002), although this may simply be due to the compressed format of the Halley VELOX data (Smith, 1995) facilitating their identification. This disparity is, however, consistent with the lower frequency of occurrence of all VLF phenomena as one goes east from Halley to SANAE-IV, which might be ascribed to the effects of the South Atlantic Anomaly. The reduced magnetic field strength in this vicinity results in the lowering of trapped particle mirror points, leading to enhanced loss through precipitation, thereby producing a diminished trapped electron population east of the anomaly.

A SCE results from the collaboration of three processes: the injection of energetic plasma into the inner magnetosphere, the eastward drift of electrons from the injection region to a ground station's field of view, and the cyclotron resonant interaction of these electrons with whistler mode waves. 
The impulsive injection of energetic particles into the nearEarth magnetotail routinely occurs at the beginning of the substorm expansion phase (e.g. McIlwain, 1974; Thomsen et al., 2001) in conjunction with heightened geomagnetic activity on the ground and in space. Two possible scenarios are generally considered to account for plasma injection: either the particles are energised in situ or they are transported from a tailward region and are energised en route. In the latter situation, injections may result from radial $\boldsymbol{E} \times \boldsymbol{B}$ drift in the inductive electric field arising from dipolarisation of the Earth's magnetic field (Li et al., 2003).

Injection events have been detected directly by geosynchronous satellites (e.g. Birn et al., 1998; Thomsen et al., 2001) and identified in energetic neutral atom images (Hendersen et al., 1997). As observed at geosynchronous orbit, injections consist of flux enhancements (by a few orders of magnitude) of particles with maximum energies up to hundreds of $\mathrm{keVs}$, and average energies of a few $\mathrm{keV}$ (Parks et al., 1980). If a satellite is located within the injection region, then the enhancement is observed simultaneously for all energies concerned and the event is "dispersionless". If, however, the injected particles only reach the satellite after drifting out of the injection region, then they become dispersed, those particles with higher energies arriving first. The location and extent of the injection region have been examined by Friedel et al. (1996), who determined that the injected particles may be distributed over a range $\pm 5 \mathrm{~h}$ around magnetic midnight and can extend inward to $L=4.3$.

The satellite observation of an injection event is reliant upon the particles being either injected directly into or drifting through the volume of space accessible to the satellite. The SCE, however, reflects the presence of injected particles within a ground station's field of view - a much larger region - and these ground-based data are thus complementary to satellite observations.

The motion of an electron, and indeed any charged particle in the magnetosphere, may be understood in terms of the conservation of the three adiabatic invariants associated with each of its periodic motions - gyration, bounce and drift (Roederer, 1970). Since the gyration and bounce of the particle occur over time scales much shorter than that associated with the development of a SCE, the motion which is of principal interest in this context is drift, which transports the particle through local time. Conservation of the invariants corresponding to gyration and bounce are nevertheless still essential to the dynamics. The drift shell, a surface generated by the motion of a particle's guiding centre, is characterised by its $L$ value and the magnetic field strength at the particle's mirror point (McIlwain, 1961). Azimuthal velocity around the drift shell is influenced by the presence of electric fields and the spatial gradient and curvature of the magnetic field.

The presence of a convection electric field directed from dawn to dusk and a corotation electric field orientated radially earthward produce an energy-independent $\boldsymbol{E} \times \boldsymbol{B}$ drift, which has both radial and azimuthal components. In the midnight-dawn quadrant this drift is directed radially earthward and azimuthally eastward.
The injected electrons are also subject to an energydependent drift as a result of the spatial gradient and curvature of the Earth's magnetic field. This drift carries them eastward, the more energetic particles and those located further from the Earth having a higher angular drift velocity (Roederer, 1970).

Subsequent to their injection, the electrons are thus transported eastward into a ground station's field of view, where they may resonantly interact with whistler mode waves.

VLF chorus is a whistler mode phenomenon encountered both in space and on the ground, consisting of numerous discrete emission elements. Particle injections are regularly correlated with enhanced chorus activity at geosynchronous orbit (Isenberg et al., 1982). Chorus is thought to be generated by the transfer of energy from anisotropic hot electrons to waves by the cyclotron resonance interaction (Sazhin and Hayakawa, 1992). Such wave-particle interactions play a role in the acceleration of electrons to relativistic energies (Meredith et al., 2001; Horne and Thorne, 2003) and induce the precipitation of energetic electrons (Horne and Thorne, 2003).

Doppler-shifted cyclotron resonance between whistler mode waves and counter-streaming electrons is described by (e.g. Hargreaves, 1992)

$W_{\|}=\frac{B^{2}}{2 \mu_{0} n_{e}} \frac{f_{B}}{f}\left(1-\frac{f}{f_{B}}\right)^{3}$,

where $W_{\|}$is the component of the electron's kinetic energy associated with motion parallel to the magnetic field, $B$ is magnetic field strength, $f_{B}$ is the electron gyrofrequency, $f$ is the frequency of the waves and $n_{e}$ is the electron number density. The interaction represented by Eq. (1) is a transverse resonance where the wave frequency is Doppler-shifted up to the electron gyrofrequency. The derivation of this relationship proceeds from the assumption that the waves are propagating parallel to the magnetic field. In general, however, the waves may possess an arbitrary wave normal angle, which, for resonance at a given frequency, leads to larger $W_{\|}$ as the direction of propagation becomes more oblique. For a predetermined population of electrons this results in a shift to higher resonance frequencies for larger wave normal angles. Within the wave generation region field-aligned propagation is favoured as this produces the greatest instability (Nunn et al., 1997).

Observational evidence suggests that chorus originates near the geomagnetic equator (Parrot et al., 2003), although recent observations of Inan (2004) indicate that the chorus source region is in rapid motion. Since the energy of gyroresonant electrons is minimised at the equator and the electron distribution is generally dominated by low energy particles, Helliwell (1965) concluded that the equatorial plane is the region in which the number of particles available for gyro-resonance is maximised and is thus the most likely location for cyclotron resonance instability. Liehmon's (1967) results, however, indicate that the off-equatorial growth rate can become quite significant, especially at higher frequencies. Regions of lower magnetic field strength and larger 
electron number density are also advantageous (Rycroft, 1972).

The penetration of whistler mode waves through the ionospheric boundary requires that the wave normal angle lie within the transmission cone (Helliwell, 1965) and consequently chorus must propagate to the ground via ducts of enhanced ionisation. The wave vectors of ducted chorus are nearly aligned with the ambient magnetic field (Parrot et al., 2003). Chorus emissions with oblique wave normals have, however, also been observed in the vicinity of the equatorial plane (Hayakawa et al., 1984) but at frequencies above half the equatorial gyrofrequency and therefore not propagating in ducted mode.

The maximum frequency for trapping of a whistler mode wave in a channel of enhanced ionisation is half the local electron gyrofrequency, $f_{B} / 2$ (Helliwell, 1965). This fact may determine the cutoff of chorus emissions at the upper edge of the SCE envelope (Smirnova, 1984). Earthward motion of electrons results in an increasing half-gyrofrequency cutoff as the particles move into regions of higher magnetic field strength. The earthward motion of electrons also produces increasing resonance frequencies, which follow from Eq. (1) as a consequence of elevated magnetic field strength and background number densities.

Wave amplification via the cyclotron resonance instability is associated with an anisotropic pitch angle distribution (Kennel and Petschek, 1966). Specifically, a pancake-shaped distribution, where large pitch angles are predominant, is unstable, while a distribution in which particle motion is primarily parallel to the magnetic field is not. Although there is a tendency for the injected plasma to be anisotropic (Birn et al., 1997) as a result of inward radial motion subject to the conservation of the first and second adiabatic invariants (Southwood and Kivelson, 1975), the subsequent dispersive drift motion of the injected electrons also leads to anisotropy in the portion of the population within a station's field of view.

The form of the resonance condition Eq. (1) implies that particles with large parallel velocities are resonant with low frequency waves and vice versa. The most energetic electrons, which arrive first within a terrestrial station's whistler mode field of view, are thus resonant with low frequency waves. Progressively higher resonant frequencies are generated as lower energy particles drift around into the field of view. This, in part, accounts for the increasing frequencies characteristic of the SCE. The frequency dispersion arising from this mechanism depends on the proximity of the station to the injection region: stations located at later local times should observe greater dispersion.

Smith et al. (1996) argued that the absence of a clear relationship between frequency dispersion and MLT in their data indicated that dispersive azimuthal drift was not the governing process in a SCE. The recent study of Abel et al. (2002) also suggests that little direct evidence exists supporting electron energy dispersion as the dominant process controlling the frequency evolution of the SCE. Neither of these publications, however, call into question whether the dispersion mechanism plays some role in the generation of the SCE phenomenon. In fact, Smith et al. (1996) proposes that the prevailing mechanism may be energy dispersion in the case of events with large frequency sweep rates and radial drift for more gradually rising events.

In this study we examine the contribution of energy dispersion in generating the frequency-time structure of the SCE by modelling the azimuthal drift of injected particles including the action of the corotation electric field. In order to isolate the effect of the gradient-curvature drift, the convection electric field is neglected and the particles are assumed to move along paths of constant $L$ in the inner magnetosphere. Although the convection field is undoubtedly of import to the dynamics, an objective of this study is to demonstrate that the inward radial $\boldsymbol{E} \times \boldsymbol{B}$ drift is not a prerequisite for SCE occurrence and that energy dispersion is a sufficient mechanism. The neglect of the convection field may be justified as follows: for $L=5$ resonance at $3 \mathrm{kHz}$ requires electrons with energies $W_{\|} \simeq 12 \mathrm{keV}$, which experience a gradient-curvature drift of $\geqslant 5 \mathrm{~km} / \mathrm{s}$. In a convection field of $0.25 \mathrm{mV} / \mathrm{m}$, representative of mildly disturbed geomagnetic conditions (Kivelson, 1976), the $\boldsymbol{E} \times \boldsymbol{B}$ drift speed at this $L$ is only $\sim 1 \mathrm{~km} / \mathrm{s}$, significantly less than the gradient-curvature drift speed. In addition, only a portion of the $\boldsymbol{E} \times \boldsymbol{B}$ drift is directed inward, while the balance contributes to the azimuthal particle drift, reducing the delay and duration of a SCE.

\section{Observations}

Examples of substorm chorus events observed at SANAE-IV $\left[71^{\circ} 40^{\prime} \mathrm{S} 2^{\circ} 50^{\prime} \mathrm{W}, L=4.3\right]$ are presented as spectrograms in Fig. 1. A selection of events are included to illustrate the variation in duration and frequency range. The broad-band VLF data were recorded remotely using the Digital VLF Recording and Analysis System (DVRAS) described by Collier and Hughes (2002). The antenna system consists of two orthogonal rhombic loops with area $58 \mathrm{~m}^{2}$ aligned geographic northsouth and east-west. The data were digitised at a sampling rate of $20 \mathrm{kHz}$.

The events on days 008,123 and 213 commence at frequencies less than $2 \mathrm{kHz}$ and proceed up to a maximum of around $6 \mathrm{kHz}$. In the interpretation of Smirnova (1984) the increase in the upper cutoff frequency over this range indicates motion of the generation region from $L=6.0$ to 4.4. The events on days 017 and 020 are confined to lower frequencies, suggesting that the source region remains at larger $L$. Furthermore, the short duration and poor dispersion of the event on day 017 indicates that the station was located close to or within the injection region and that the injected particles were not initially distributed over a very large range of local times.

The event on day 020 is particularly interesting as there is clear evidence of three drift echoes. An isolated injection of particles was detected by geosynchronous satellite 1990 095 at 03:50 UT. A weak SCE was subsequently recorded at SANAE-IV, starting just before 04:00 UT, followed by 


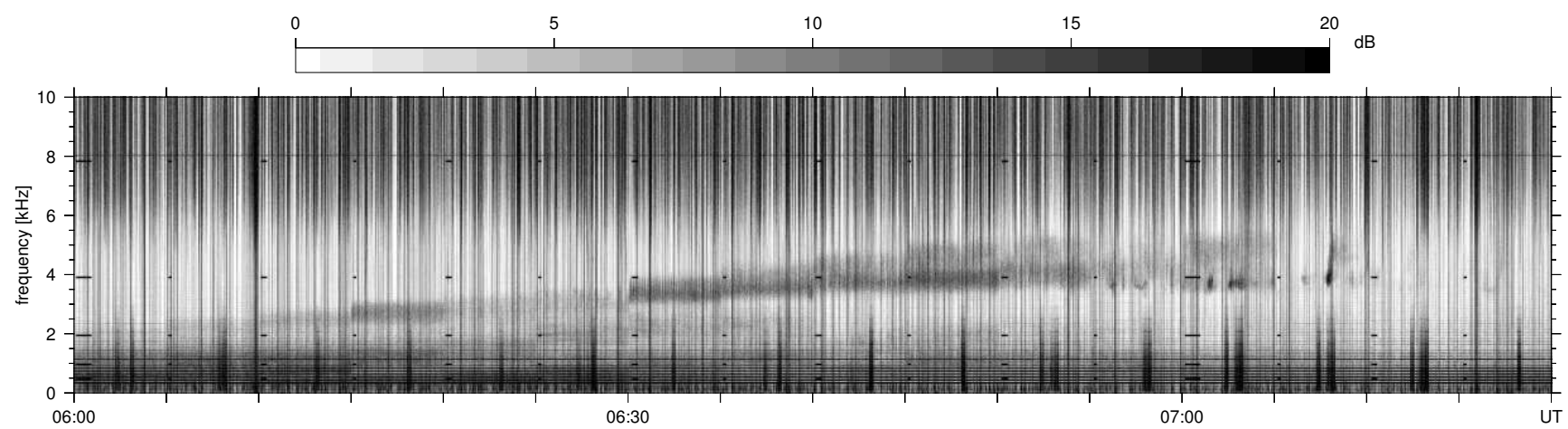

(a) 2002 day 008

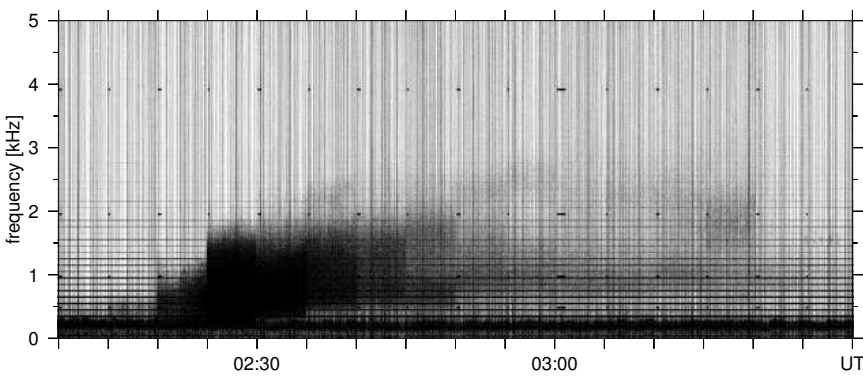

(b) 2002 day 017

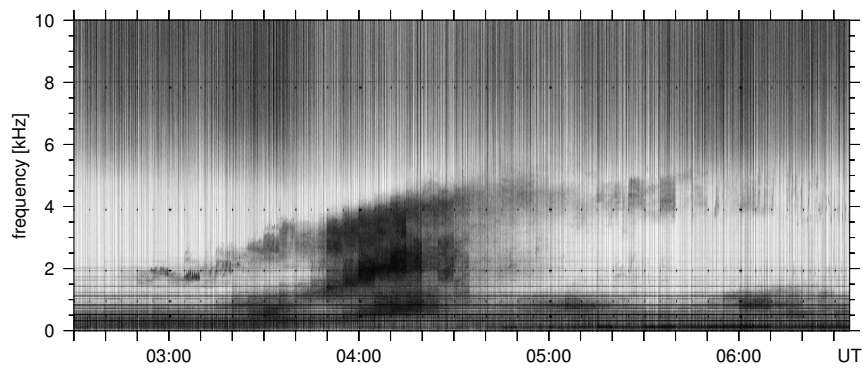

(d) 2002 day 123

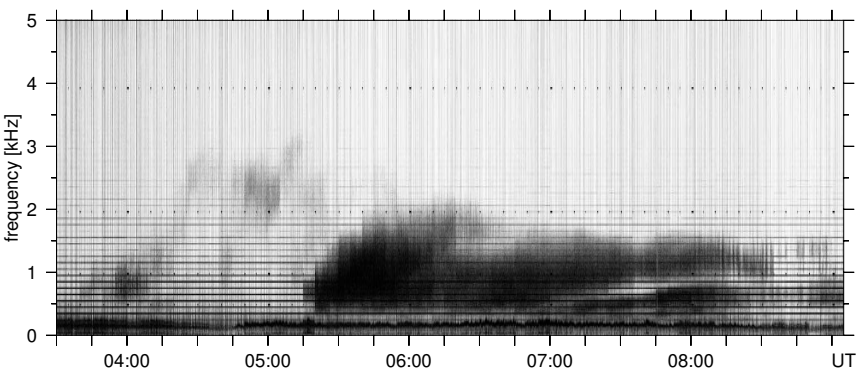

(c) 2002 day 020

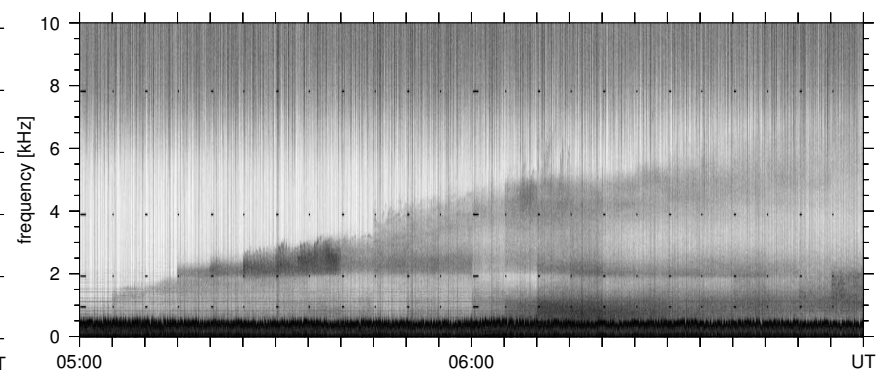

(e) 2002 day 213

Fig. 1. Broad-band VLF data from SANAE-IV with examples of substorm chorus events. Each panel is a spectrogram representing the intensity of the signal as a function of frequency and time. Data were recorded in synoptic mode, with only one in every five minutes sampled.

Table 1. Drift periods ( $\mathrm{min}$ ) in corotating frame for particles with equatorial pitch angle $\alpha=45^{\circ}$ in a dipole field.

\begin{tabular}{ccc}
\hline$W_{\|}[\mathrm{keV}]$ & $L=5$ & $L=6$ \\
\hline 100 & 56.1 & 46.7 \\
200 & 30.9 & 25.7 \\
300 & 22.0 & 18.3 \\
\hline
\end{tabular}

three stronger events at intervals of roughly $1 \mathrm{~h}$ measured at $500 \mathrm{~Hz}$. Each of the echoes was observed to have a progressively larger frequency dispersion. Resonance at $500 \mathrm{~Hz}$ for $L$ between 5 and 6 is achieved by particles with $W_{\|}$in the range 100 to $300 \mathrm{keV}$. Typical drift periods for such particles are given in Table 1.
The range of frequencies observed on the ground is characteristic of the conditions prevailing in the magnetospheric source region. Extensive propagation in the Earthionosphere waveguide should, however, result in waveguide cutoffs (Helliwell, 1965) becoming apparent. The event of day 213 has a definite reduction in emission intensity at frequencies below $\sim 2 \mathrm{kHz}$, consistent with waveguide cutoff under an ionosphere at an altitude of $75 \mathrm{~km}$. This cutoff is not evident in the other SCE and one may thus conclude that these enter the waveguide in closer proximity to the station.

The events in Fig. 1 are also apparent in the Halley $\left[75^{\circ} 35^{\prime} \mathrm{S} 26^{\circ} 45^{\prime} \mathrm{W}, L=4.4\right]$ VELOX data, examples of which are given in Fig. 2. Halley is located around $1.5 \mathrm{~h}$ west of SANAE-IV, which may account for the differences in the frequency sweep rate and the duration of events observed at both stations. 


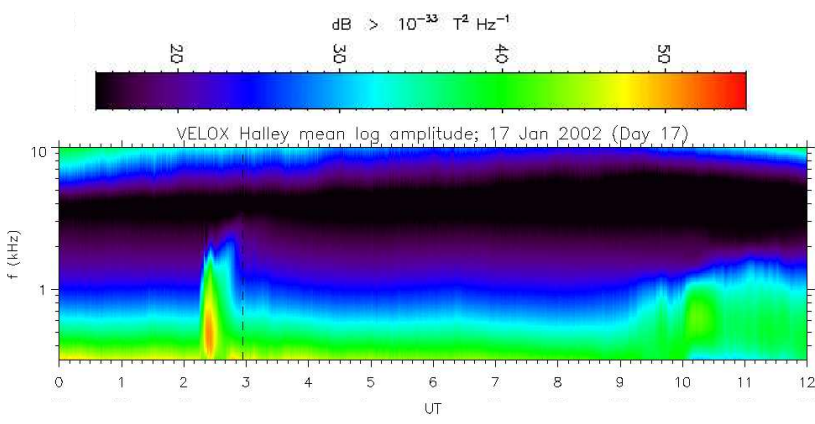

(a) 2002 day 017

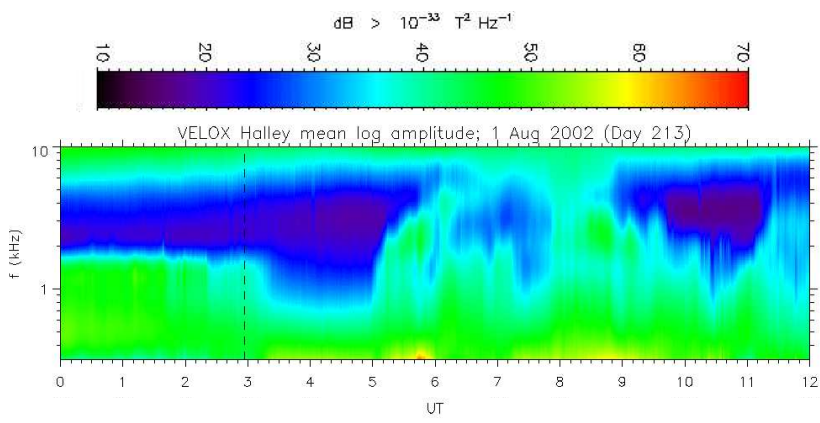

(b) 2002 day 213

Fig. 2. Halley VELOX data corresponding to SANAE-IV SCE presented in Figs. $1 \mathrm{~b}$ and e.

Each of the events presented in Fig. 1 is accompanied by particle injections detected at geosynchronous orbit. Electron flux data from Synchronous Orbit Particle Analyser (SOPA) instruments on board LANL geosynchronous satellites are displayed in Fig. 3, where it is evident that injections result in a dramatic increase in the flux of particles with energies of tens to hundreds of keV. Satellite 1990-095 is located closest to the meridian of SANAE-IV, on a field line $9^{\circ}$ west of the station, and should thus detect particle injections before they manifest themselves as SCE at SANAE-IV.

In Fig. 3a an injection of electrons is recorded on satellite $1990-095$ at 02:13 UT, when the satellite is ideally situated in the vicinity of local midnight. This injection is a "near-dispersionless substorm onset" (Friedel et al., 1996) as particles of all energies arrive at the satellite almost simultaneously. The duration of the injection is short - comparable to the time scale of the magnetic field dipolarisation - but an elevated electron flux is sustained for a longer period as a result of the drift of electrons injected to the west of the satellite. The rapid flux enhancement is followed within $7 \mathrm{~min}$ by the onset of chorus emissions at SANAE-IV.

The SOPA data for day 123 in Fig. 3b indicates a minor injection at 03:16 UT followed by a more significant one at 03:42 UT. Some time later the injected particles are detected by satellites LANL-97A, 1994-084 and 1991-080, respectively, where the dispersive effects of the gradientcurvature drift are apparent. Careful examination of the VLF data in Fig. 1d reveals that chorus activity starts at around 03:00 UT and a second band of emissions begins at

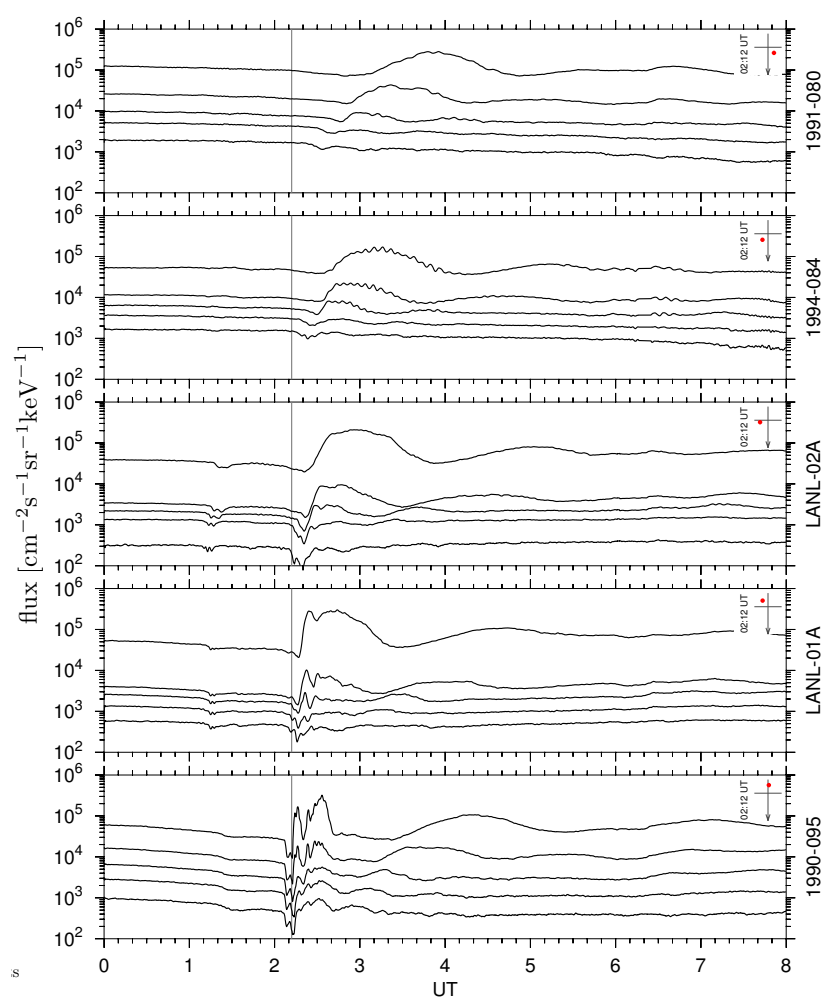

(a) 2002 day 017

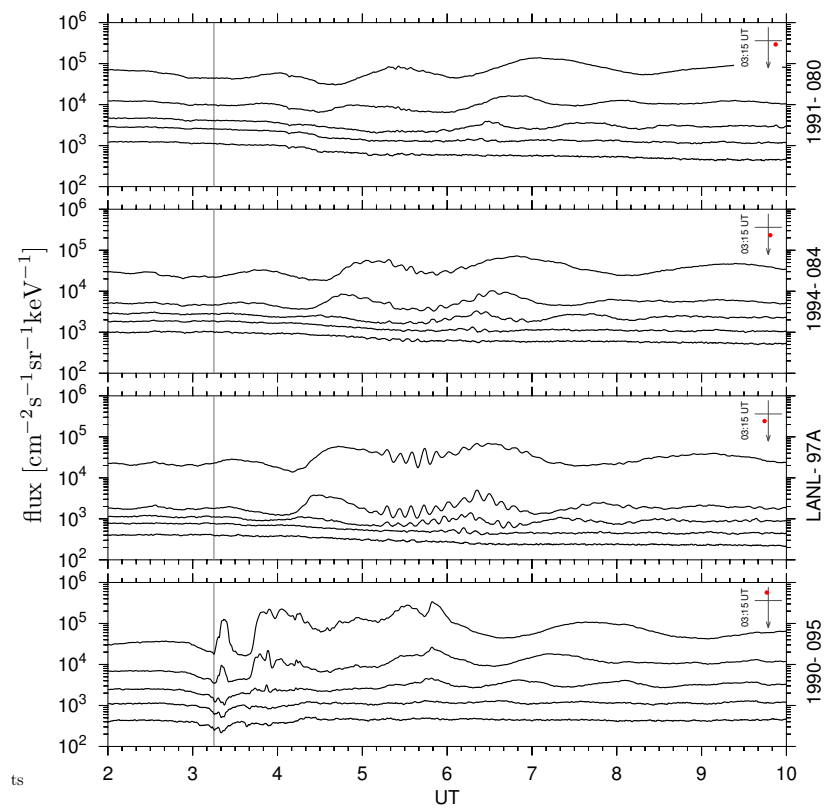

(b) 2002 day 123

Fig. 3. Electron flux data from SOPA instruments on LANL geosynchronous satellites for SCE presented in Figs. 1b and d. The five traces represent different energy channels; from top to bottom they are $50-75,75-105,105-150,150-225$ and $225-315 \mathrm{keV}$. In the upper right corner of each panel is an inset indicating the location of the satellite at approximately the moment of injection (the arrow points sunward). 


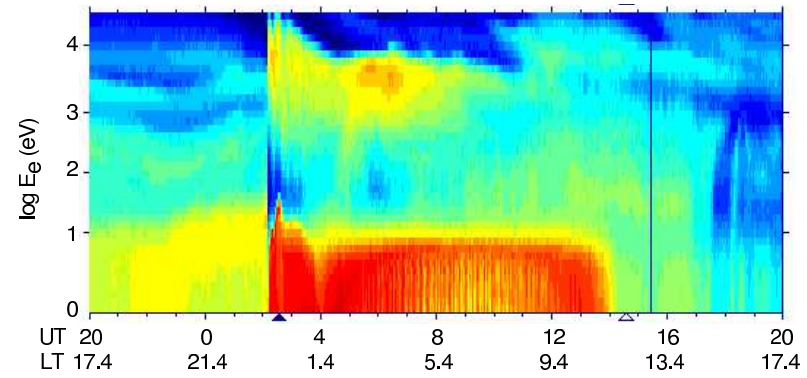

(a) 2002 day 017

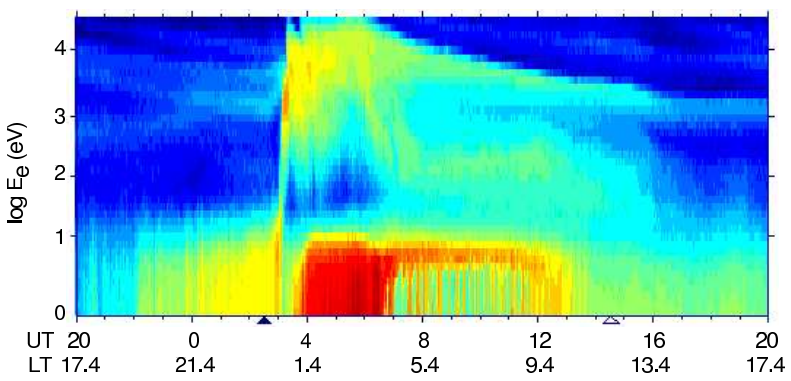

(b) 2002 day 123

Fig. 4. Low energy MPA electron data from spacecraft 1990-095 for periods corresponding to the SCE presented in Figs. $1 \mathrm{~b}$ and d.

about 03:40 UT. In both cases the onset of the VLF activity precedes the geosynchronous injection, a somewhat surprising result, suggesting that this injection was centred in the midnight-dawn quadrant or that the VLF activity was initiated at $L>6.6$.

The bulk of the SCE emissions are generated by particles with energies lower than those detected by the SOPA instrument. The Magnetospheric Plasma Analyser (MPA) data in Fig. 4 reflects the low energy component of the injected particles, indicating a substantial increase in the flux of electrons with energies of a few to tens of $\mathrm{keV}$. The SOPA and MPA data also illustrate the existence of upper and lower energy cutoffs in the spectrum of injected electrons (Reeves, 1998).

The onset of the substorm expansion phase is reflected in magnetic perturbations observed both on the ground and in orbit. The substorm AE index is a measure of the strength of the auroral electrojet, and as such, provides an indicator of substorm activity. Although neither provisional nor final $\mathrm{AE}$ data is presently available for 2002, quicklook data indicate substorm signatures in the $\mathrm{AE}$ and related indices at the appropriate times for all the events in Fig. 1.

The results of Smith et al.'s (1999, 2002) superposed epoch analysis register negative and positive bays, respectively, in the $H$ and $D$ magnetic field components recorded by the Halley magnetometer in association with substorm onset and coincident with the observation of a SCE. Halley is normally somewhat equatorward of the auroral oval around midnight, and negative $H$ bays are consistent with a westward horizontal electrojet poleward of a Southern Hemisphere station at sub-auroral latitudes. The SCE's considered by Smith et al. (1999) were selected with the intent of study-
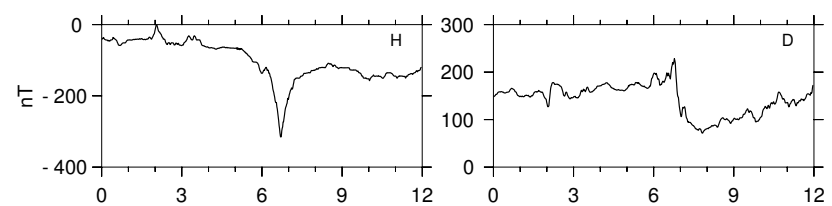

(a) 2002 day 008

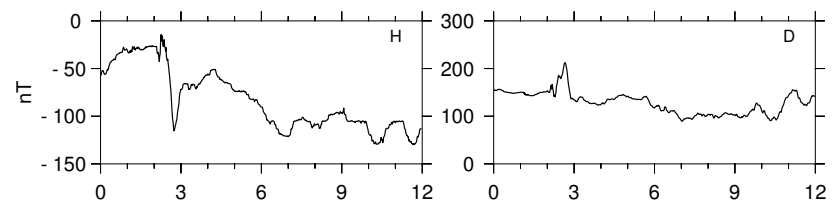

(b) 2002 day 017

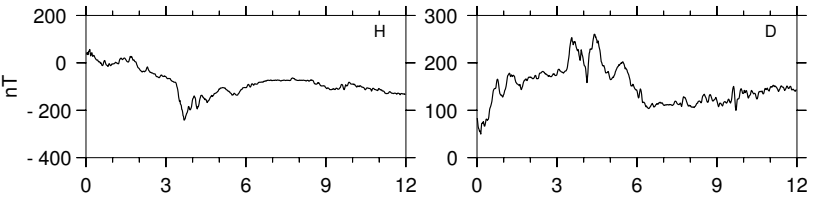

(c) 2002 day 020

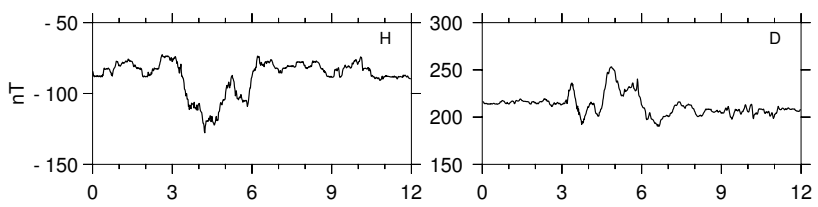

(d) 2002 day 123

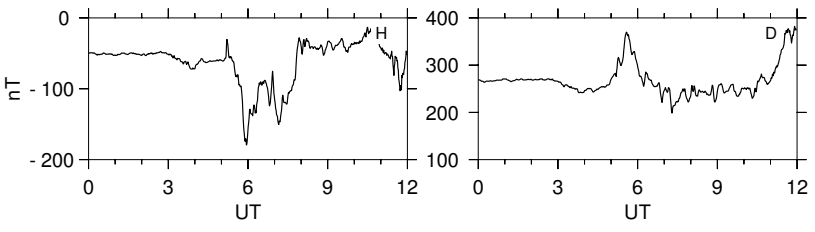

(e) 2002 day 213

Fig. 5. Variation of the $H$ and $D$ magnetic field components recorded by the Halley fluxgate magnetometer for the events displayed in Fig. 1.

ing their relationship with the substorm current wedge. Considering only SCE recorded at Halley within one hour of local midnight, Smith et al. (1999) found that the start of the $D$ bay was simultaneous with the commencement of the SCE, while the $H$ bays started before the SCE appeared but exhibited a change in gradient close to the SCE epoch. The average magnitudes of the bays examined by Smith et al. (1999) were $\delta H=-140 \mathrm{nT}$ and $\delta D=+65 \mathrm{nT}$.

Data presented in Fig. 5 represent $H$ and $D$ magnetic field variations at Halley for the periods corresponding to the events in Fig. 1. In each case $H$ and $D$ bays of the correct sense were discernible at a time indicating their association with the corresponding SCE and providing additional evidence that the SCE is consequent to substorm onset.

The cyclotron wave-particle interaction results in a decrease in the pitch angle of the resonant electrons and may lead to their precipitation (Foster and Rosenberg, 1976; Rosenberg et al., 1981). Electron precipitation can be monitored using a riometer, which indicates the degree of absorption of cosmic VHF radio noise due to charged particle den- 


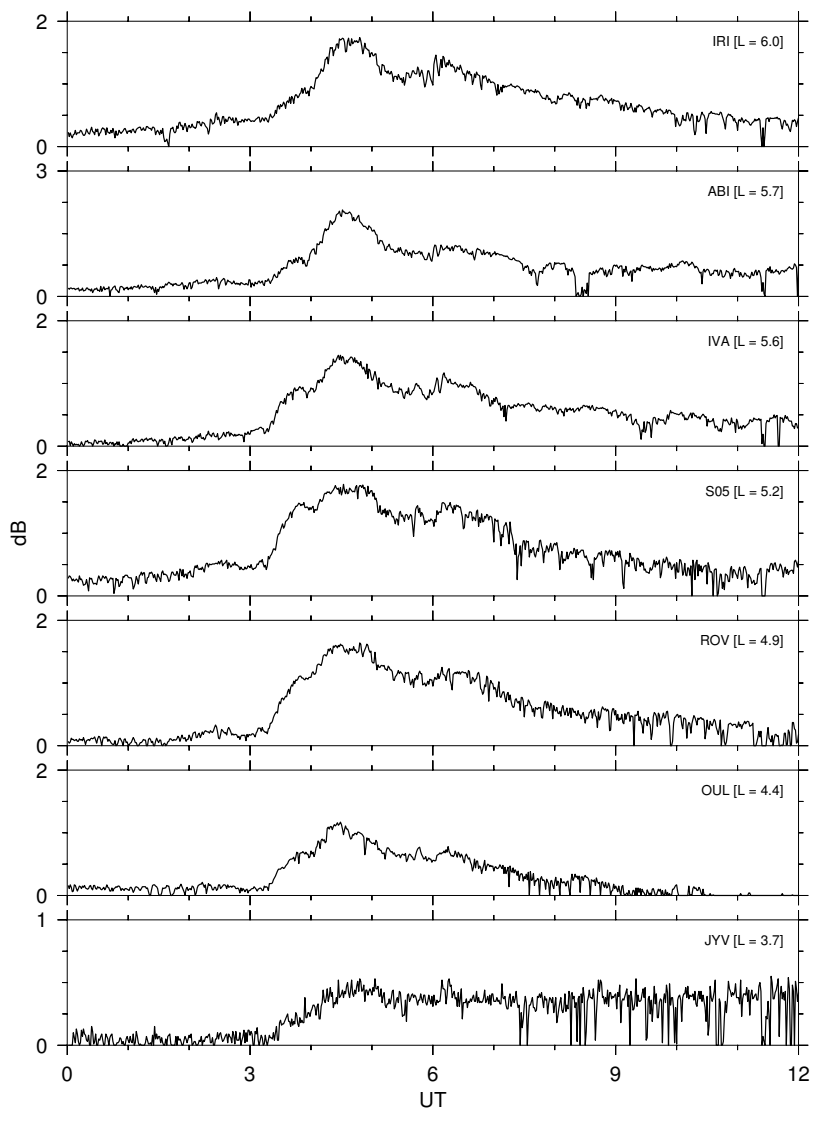

Fig. 6. Data from the Nordic riometer chain for 2002 day 123, indicating enhanced electron precipitation associated with the SCE in Fig. 1d.

sity variations in the ionosphere. Data from the Nordic chain of riometers for 2002 day 123 are presented in Fig. 6. The degree of absorption recorded by these instruments increases significantly just after 03:00 UT, which corresponds to the onset of the SCE in Fig. 1d. The precipitation is most evident for stations located outside the plasmapause, but is still discernible at JYV (Jyväskylä), which lies on a field line within the plasmasphere and OUL (Oulu), which is situated close to the plasmapause. The fact that enhanced precipitation is observed at stations located over the range $L=3.7$ to 6.0 indicates that wave-particle interactions occur over the corresponding region in the magnetosphere.

It should be stated that the data in Fig. 6 is a particularly fine example. Such a well-defined precipitation enhancement is not always observed in conjunction with a SCE, or it is detected at only a subset of the riometer stations.

\section{Model}

Consider a simple model based on the geometry illustrated in Fig. 7, with the injection of energetic particles taking place over a range of local time, $\delta \psi$, centred on midnight and an observer located at local time $\psi_{0}$ at the moment of injection.

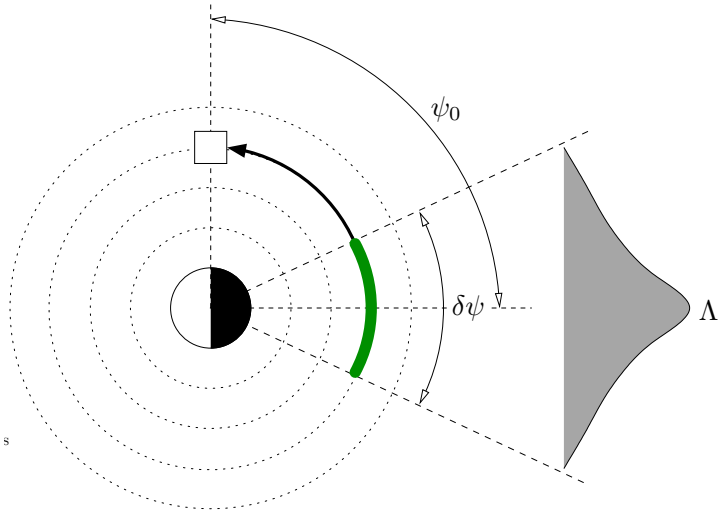

Fig. 7. Geometry of the model: injection over range of local time, $\delta \psi$, centred on midnight (green curve) and an observer (open box) located at local time $\psi_{0}$ at the moment of injection. The distribution of the injection intensity in local time is represented by the shaded region.

The observer may be situated either in the magnetosphere or at a terrestrial station, where, in the latter case, propagation to the observer is assumed to take place along ducts of augmented plasma density. In practice, the injected particles would be distributed over a range of $L$; however, this model considers azimuthal drift alone and so we confine our attention to those particles originating on a particular $L$ shell.

In addition, for the reasons cited in Sect. 1 we further constrain the model to examine only the distribution of particles in the equatorial plane.

The drift motion of the injected particles is calculated in a dipole magnetic field, where the assumption of a dipolar geometry is not unreasonable since particle injection accompanies relaxation of the field. The effects of a convection electric field are neglected but corotation drift, arising from the azimuthally symmetric corotation electric field, is implicitly taken into account as the particle trajectories are calculated in the Earth's rotating frame of reference. Although the electron dynamics are treated here in a simplified model of the magnetosphere, results for more realistic field geometries are to be found elsewhere (e.g. Reeves et al., 1991).

Suppose that the source of particles at local time $\psi$ and time $t$ is described by

$S(\psi, t ; W, \alpha)=\Lambda(\psi) \delta(t) F(W, \alpha)$,

where $\Lambda$ describes the local time distribution of the source and $F$ its energy and pitch angle dependence. The time impulse in Eq. (2) is justified by the fact that the duration of the injection event is short compared with the time scale of the evolution of the SCE. However, in principle, the distribution function resulting from a source with arbitrary temporal structure may be derived from that ensuing from an impulsive source by convolution.

The particle population is assumed to consist of a background low-temperature thermal component with density $n_{c}$, 


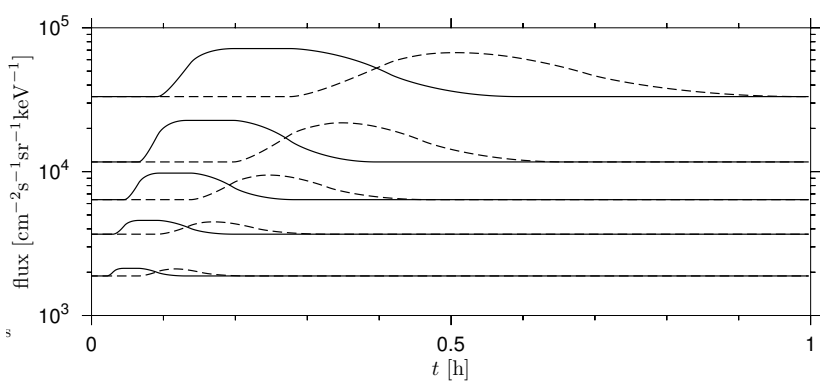

(a) uniform source, $\Lambda=\Lambda_{1}$

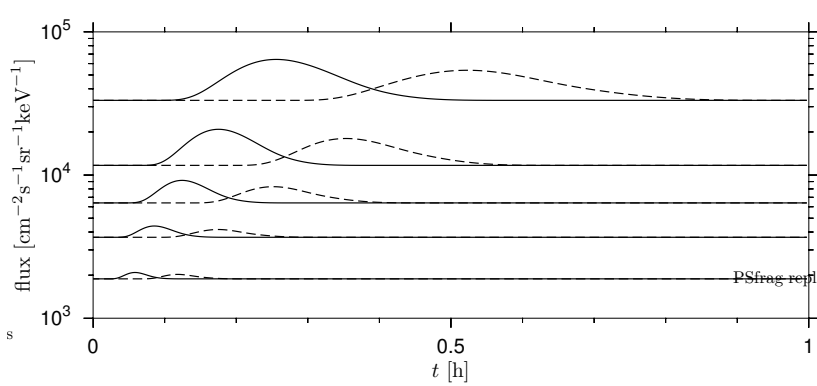

(b) peaked source, $\Lambda=\Lambda_{2}$

Fig. 8. Simulated flux signatures at geosynchronous orbit, three (solid line) and six (dashed line) hours east of midnight. Injections distributed in local time according to $\Lambda_{1}$ and $\Lambda_{2}$. Energy channels are the same as in Fig. 3.

and a high-temperature component with density $n_{h}$ and distribution $F$, normalised such that

$n_{h}=2 \pi \int_{0}^{\pi / 2} \int_{0}^{\infty} F(W, \alpha) v^{2} \sin \alpha d v d \alpha$

The total number density in the injection region is $n_{e}=n_{c}+n_{h}$, where $n_{h} \ll n_{c}$ and consequently $n_{e} \simeq n_{c}$ (Liemohn, 1967). The injection intensity may be estimated using the LANL flux data, from which $n_{h} \sim 10^{5} \mathrm{~m}^{-3}$ is appropriate. Only a small fraction of the injected hot plasma density is present at the observer at any given time and it is assumed that only the non-thermal particles participate in the resonant interactions.

The functional forms which should be adopted for $\Lambda$ and $F$ are somewhat uncertain. Experimental evidence suggests that the region into which particles are injected is centred on midnight (Birn et al., 1997; Thomsen et al., 2001); however, little appears to be known about the variation of the injection intensity as a function of local time. Selecting $\delta \psi=45^{\circ}$ produces an injection region spanning three hours of local time, consistent with the results of Reeves et al. (1991), although the extent of this region may vary appreciably (Reeves et al., 1992). The simplest assumption is that the particles are uniformly distributed:

$\Lambda_{1}(\psi)=\Pi(\psi / \delta \psi)$, where $\Pi$ is the rectangle function (Bracewell, 1965). Another option is to introduce a peak in the local time distribution of the source:

$\Lambda_{2}(\psi)=\Pi(\psi / \delta \psi) \cos ^{m}(\pi \psi / \delta \psi)$.

Setting $m=2$ provides a peaked distribution which goes smoothly to zero at its extremities. The flux signatures expected from injections conforming to $\Lambda_{1}$ and $\Lambda_{2}$ are given in Fig. 8, where it is apparent that the former is more consistent with the observations in Fig. 3.

The kinetic distribution of the injected plasma may be represented by a superposition of Maxwellians with different characteristic temperatures (Parks et al., 1980), and consequently we consider a source spectrum described by a biMaxwellian distribution

$F_{1}(W, \alpha)=n_{h} k_{1} \exp \left[-W\left(\frac{\sin ^{2} \alpha}{T_{\perp}}+\frac{\cos ^{2} \alpha}{T_{\|}}\right)\right]$,

where $k_{1}=\sqrt{m^{3} / 2 \pi^{3} T_{\perp}^{2} T_{\|}}$, characterised by temperatures $T_{\perp}$ and $T_{\|}$, both of which have units of energy.

Measurements used to derive phase space densities indicate that a power law function of energy may be a more appropriate representation, especially at larger energies (e.g. Birn et al., 1997). The generalised Lorentzian distribution (Vasyliūnas, 1968)

$F_{2}(W, \alpha)=n_{h} k_{2}\left(1+\frac{W}{W_{0}}\right)^{-(\kappa+1)}$,

where $\kappa$ is the spectral index and $W_{0}$ is related to the effective temperature by $W_{0}=(\kappa-3 / 2) T$, may be used to model a plasma with a non-Maxwellian high-energy tail. The normalisation factor has the form

$k_{2}=\frac{1}{\sqrt{2}}\left(\frac{m}{\pi W_{0}}\right)^{3 / 2} \frac{\Gamma(\kappa+1)}{\Gamma(\kappa-1 / 2)}$.

Li et al. (2003) used $\kappa=1.8$ while Birn et al. (1998) had $\kappa=2.5$; both set $T=0.5 \mathrm{keV}$. The results quoted here are not especially sensitive to $\kappa$, and consequently an intermediate value of $\kappa=2$ is employed. The isotropic distribution Eq. (7) may be modified by introducing a factor dependent on pitch angle, $\sin ^{2 p} \alpha$. The data of Maeda and Lin (1981) suggest that values of $p$ up to 1 are reasonable.

In the absence of a dawn-dusk electric field, the injected electrons undergo eastward gradient-curvature drift along paths of constant $L$. Particles with energy $W$ and pitch angle $\alpha$ acquire a bounce-averaged equatorial azimuthal drift velocity of (Roederer, 1970)

$\dot{\psi}=\frac{3 W}{q B_{0} R_{\mathrm{E}}^{2}} L G(\alpha)$,

where $R_{\mathrm{E}}$ is the Earth's radius, $B_{0}=30.1 \mu \mathrm{T}, q$ is the charge of an electron and, to reasonable approximation,

$G(\alpha)=0.70+0.30 \sin \alpha$ for $40^{\circ} \lesssim \alpha \leqslant 90^{\circ}$. 
A better approximation for $G(\alpha)$, accurate for small $\alpha$, may be found in Ejiri (1978); however, the simpler form Eq. (9) is employed for the calculations presented here. The phase space density of particles at the observer, $g(W, \alpha, t)$, can be derived from Eq. (2): following Jentsch (1976, Eq. 5), one has at time $t$,

$g(W, \alpha, t)=\Lambda\left(\psi_{0}-\int_{0}^{t} \dot{\psi} d t^{\prime}\right) F(W, \alpha)$.

The contribution of particles that have made multiple orbits around the Earth may be consolidated in Eq. (10) by taking the argument of $\Lambda$ modulo $2 \pi$.

The growth rate of whistler mode waves of frequency $f$ in resonance with electrons having parallel velocity $v_{\|}=v_{\mathrm{R}}$ was found by Kennel and Petschek (1966) to be

$\gamma=2 \pi^{2} \eta f_{B}\left(1-\frac{f}{f_{B}}\right)^{2}\left(A-A_{c}\right)$,

where the anisotropy, in the form given by Etcheto et al. (1973), is expressed as

$$
A=\left.\frac{\int_{0}^{\pi / 2} \frac{\partial g}{\partial \alpha} \tan ^{2} \alpha \sec ^{2} \alpha d \alpha}{2 \int_{0}^{\pi / 2} g \tan \alpha \sec ^{2} \alpha d \alpha}\right|_{W=W_{\|} \sec ^{2} \alpha}
$$

and

$$
\eta=\left.\frac{2 \pi v_{\mathrm{R}}^{3}}{n_{e}} \int_{0}^{\pi / 2} g \tan \alpha \sec ^{2} \alpha d \alpha\right|_{W=W_{\|} \sec ^{2} \alpha}
$$

describes the relative number of particles in resonance. In Eqs. (12) and (13) $g$ is evaluated subject to the condition $W=W_{\|} \sec ^{2} \alpha$, where $W_{\|}$is the resonant energy determined by Eq. (1). As the resonant energy is related to the wave frequency through the resonance condition, both $A$ and $\eta$ are also functions of frequency. These quantities are calculated on the observer's meridian and the longitudinal extent of the observer's field of view is ignored. Under conditions of azimuthal symmetry, a finite field of view could be accounted for by appropriately broadening the injection region.

The assumption $n_{h} \ll n_{c}$ allows for the use of $n_{c}$ instead of $n_{e}$ in the calculation of $\eta$, which is computationally expedient, as one does not have to determine the total density of the hot plasma component at the observer. However, the validity of this supposition is questionable, as with increasing $L$ the density of the two populations may become comparable.

Instability of whistler mode waves depends on the anisotropy exceeding a critical value,

$$
A_{c}=\frac{1}{f_{B} / f-1} .
$$

Consequently, for a distribution with $A=1$, only waves with frequencies less than half the gyrofrequency are unstable. Since $A_{c}$ increases as $f \rightarrow f_{B}$, progressively more anisotropic distributions are required to produce instability at frequencies approaching the electron gyrofrequency. Spacecraft observations of chorus emissions above half the local electron gyrofrequency (e.g. Meredith et al., 2001) therefore either imply that they were triggered by a highly anisotropic population of electrons or that they originated in an offequatorial region of higher magnetic field strength.

An isotropic distribution attenuates waves at all frequencies, but there always exists a range of frequencies which are amplified by a distribution with $A>0$ (Liemohn, 1967). If the distribution function is a bi-Maxwellian, then the anisotropy is $A=T_{\perp} / T_{\|}-1$. If the angular dependence of the density function is of the form $\sin ^{2} p$, then the anisotropy is simply $A=p$. However, since in the model considered here the distribution function at the observer at a given time is determined by those particles that drift from the source region to the observer in the time interval elapsed since injection, neither of these simple cases emerge.

If the distribution function, $g(W, \alpha, t)$, is independent of pitch angle, or isotropic, then the anisotropy is identically zero. The function $\Lambda$ enters into Eq. (10) as a weighting factor which depends on the azimuthal drift velocity and time. Although the drift velocity is a function of $\alpha$, it serves only to determine the location in the source region from which an electron originates, and hence the applicable weight. In order to obtain a non-isotropic distribution at the observer, $\partial g / \partial \alpha \neq 0$, it is necessary that either $\partial F(W, \alpha) / \partial \alpha \neq 0$ or $d \Lambda / d \psi \neq 0$ within the source region. The fact that the distribution at the observation point evolves with time as particles drift around from the injection region implies that if the source distribution is not isotropic or the source particles are not distributed uniformly in local time then the observed anisotropy is non-zero and also varies with time.

In principle, the calculated growth rate and a knowledge of the background embryonic wave amplitudes, combined with processes limiting the exponential wave growth, could be used to estimate the absolute wave amplitudes resulting from this interaction. Kennel and Petschek (1966) showed that the resonant particle distribution is driven towards a state of marginal stability as a result of its interaction with the waves. Modification of the electron population arising from wave amplification would lead to a reduction in the pitch angles of interacting particles, resulting in those particles close to the loss cone being precipitated into the upper atmosphere. For the purposes of this model the wave energy density is assumed to be significantly smaller than that of the particles and, consequently, action of the waves on the resonant particle distribution is neglected. The validity of this approximation is somewhat uncertain as there is compelling evidence (enhanced precipitation in riometer data) that the particle distribution is indeed modified during the interaction. Although imperfect we adopt this approximation as it results in substantial computational simplifications. 


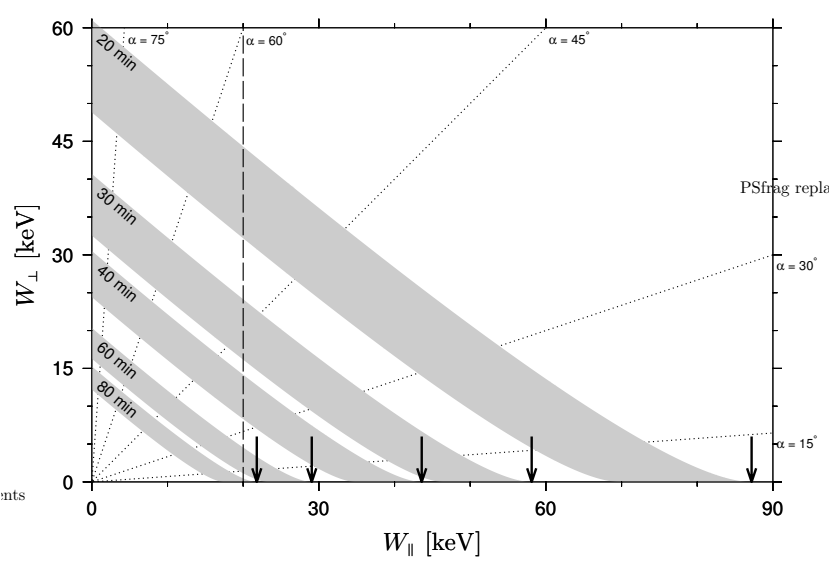

Fig. 9. Components of the particle energies at the observation point. The shaded regions represent the range of $W_{\perp}$ and $W_{\|}$occupied by particles for $t=20,30,40,60$ and $80 \mathrm{~min}, \delta \psi=10^{\circ}$ and $\psi_{0}=45^{\circ}$ at $L=5$. The arrows indicate the cutoffs in $W_{\|}$at each of these times. Dotted lines are curves of constant pitch angle.

\section{Results}

The whistler mode field of view for satellite observations is restricted and therefore, using either models or observations of the plasma density and magnetic field strength, one is able to derive a unique mapping between the observed wave frequency and the resonant electron energy (e.g. Abel et al., 2002). In contrast, ground stations are able to detect waves originating over a large range of $L$ and this introduces ambiguity into the relationship between the observed frequency and the resonant electron energy.

The whistler mode field of view for a ground station at $L \approx 4$ may be estimated as around $30^{\circ}$ in longitude and $L$ roughly between 3 and 7 (Carpenter, 1966; Smith et al., 1996). The majority of the results quoted here are for injections at $L=5$. This particular value was selected as it falls in the middle of the aforementioned range of $L$ and, furthermore, this represents the average location of Mauk and McIlwain's (1974) injection boundary for moderate $K_{p}$. Although the simulations of $\mathrm{Li}$ et al. (1998) indicate that the injection boundary model is not necessary to explain dispersionless injections, it serves here as an approximation to the locus of the injected particles.

The radial electron density profile used is the empirical model of Carpenter and Anderson (1992). For mildly disturbed magnetospheric conditions, representative of those prevailing during the events in Fig. 1, one has in the morning sector $n_{c}=4.5 \times 10^{6} \mathrm{~m}^{-3}$ at $L=5$, while the plasmapause is located at $L \simeq 4$.3. The injected plasma is presumed to have $n_{h}=10^{5} \mathrm{~m}^{-3}$.

The shaded regions in Fig. 9 represent the relationship between $W_{\perp}$ and $W_{\|}$for electrons that have the drift rate required to reach the observer at a selection of times after the moment of injection. Only electrons which have not made an entire orbit around the Earth are considered. The higher and lower energy borders of the shaded regions correspond to electrons injected at the two extremities of the source region, the upper boundary being associated with those particles originating at the edge most remote from the observer. The asymmetry between $W_{\perp}$ and $W_{\|}$for the observed population is accounted for by the fact that for smaller pitch angles a higher energy is required to achieve the same drift rate. The form of these regions places an upper bound on $W_{\|}$(indicated by the arrows) for a given $t$, which, in turn, places a lower bound on the resonant frequency. As time proceeds the distribution of particles at the observer shrinks towards the origin. This implies an upper cutoff in $W_{\|}$that decreases with time, which leads to an increasing lower cutoff in the resonance frequency.

This lower frequency cutoff determines the latest time at which amplification at a given frequency ceases. However, if the anisotropy falls below the critical level before that, then wave generation will terminate sooner. In practice, the anisotropy becomes sub-critical before $\eta \rightarrow 0$.

It is informative to explore the influence that the form of the source distribution function exerts on the magnitude and evolution of the anisotropy at the observer. The integrands in the numerator and denominator of Eq. (12) are plotted as a function of $\alpha$ for two different source models in Fig. 10, reflecting the variation in these functions along the dashed line in Fig. 9. The shaded rectangles represent the range of $\alpha$ for which the functions are non-zero at the indicated time, taking into account the width of the source and the travel time from the source region to the observer. Within the range of pitch angles present at the observer at a given instant, the larger $\alpha$ correspond to particles arriving from the furthest part of the injection region. This is due to the fact that for a given value of $W_{\|}$, both $W$ and $G(\alpha)$, and hence the azimuthal drift velocity, increase with $\alpha$.

For $\Lambda=\Lambda_{1}$ one must have $\partial F / \partial \alpha \neq 0$ to achieve non-zero anisotropy. However, if $\Lambda$ is a non-uniform function of $\psi$ then finite anisotropies are found at the observer even if $F$ is independent of $\alpha$. In Fig. 10 the bell-shaped curves for the denominator reflect the effect of the non-uniform $\Lambda=\Lambda_{2}$ distribution of the source in azimuth. This local time distribution introduces a bipolar shape to the gradient curves at the observer, arising primarily from the derivative of $\Lambda_{2}$ with respect to $\psi$. Since both source distributions considered are isotropic, the asymmetry between the two lobes of the bipolar curve originates not only from the trigonometric factors but also from the dependence of $F$ on $W$. Specifically, the distribution of particles from a Maxwellian injection declines far more swiftly with increasing $\alpha$ along a line of constant $W_{\|}$than that resulting from a Lorentzian injection. Consequently, the difference in the area under the two lobes of the curve representing the numerator of Eq. (12) is larger in the case of the Maxwellian and the resulting anisotropy is accordingly greater. This is exemplified by the data plotted in Fig. 11 which illustrate that the anisotropy produced by a Maxwellian injection is initially very large but is reduced rapidly, while that resulting from a Lorentzian injection is smaller but dwindles more gradually with time. 


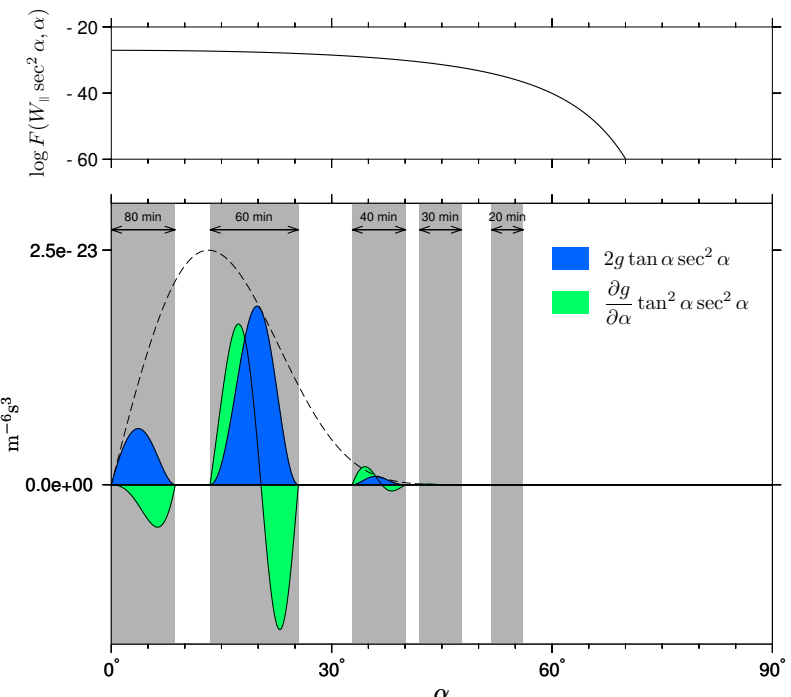

(a) Maxwellian $(T=2 \mathrm{keV})$

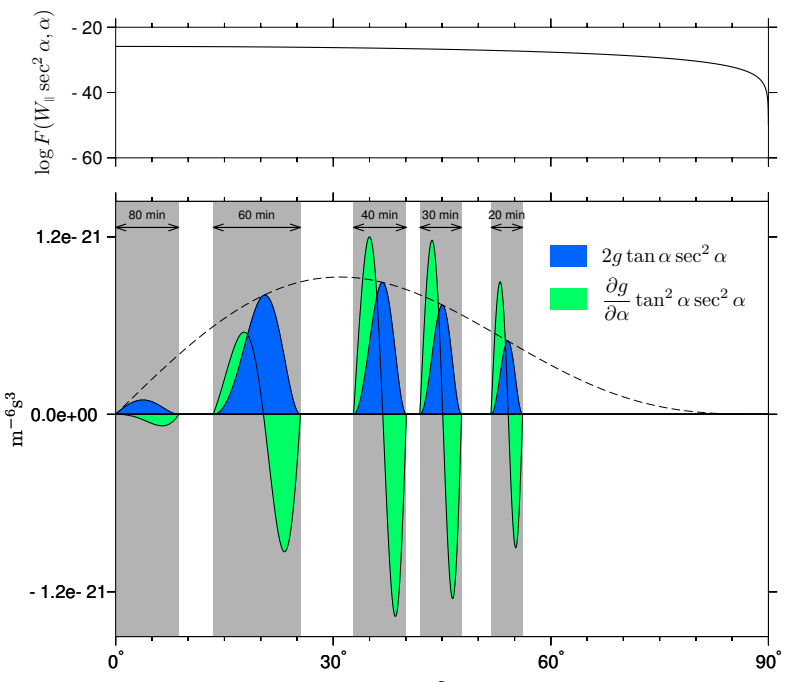

(b) Lorentzian ( $T=2 \mathrm{keV}, \kappa=2$ and $p=0)$

Fig. 10. Integrands in the numerator and denominator of Eq. (12) evaluated along the dashed line in Fig. 9, for $W_{\|}=20 \mathrm{keV}$ with $\Lambda=\Lambda_{2}$. The dashed curves represent the variation of the source density function while solid curves reflect the relationship for the density function at the observer at selected times. A narrow source region, $\delta \psi=10^{\circ}$, is employed to prevent overlap of the curves for different times.

In fact, the isotropic Lorentzian produces an anisotropy which is always below the critical value and consequently, does not lead to wave growth. However, setting $p=1$ for the Lorentzian distribution enhances the proportion of particles with larger pitch angles and results in an anisotropy which is initially in excess of $A_{c}$.

\subsection{Maxwellian source}

Consider first a bi-Maxwellian source distribution. The growth rate as a function of time for a range of resonance fre-

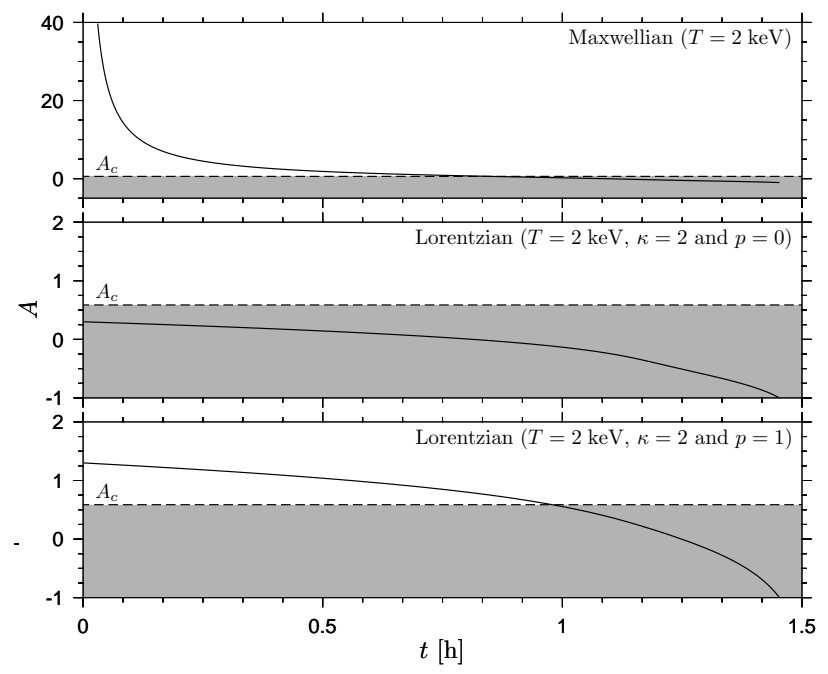

Fig. 11. Evolution of the anisotropy $\left(W_{\|}=20 \mathrm{keV}\right)$ at $\psi_{0}=45^{\circ}$ and $L=5$, for various forms of the source distribution function.

quencies at an observer located in the midnight-dawn quadrant, $\psi_{0}=45^{\circ}$, is plotted in Fig. 12a. Since in this case the injection is uniformly distributed over $\delta \psi$, it is mandatory that the source be inherently anisotropic $\left(T_{\perp} \neq T_{\|}\right)$. The uppermost panels are greyed out as these lie above half the equatorial electron gyrofrequency and are consequently not observed on the ground. The peaks in growth rate at successively higher frequencies occur at progressively later times, in agreement with the observed SCE behaviour.

In Fig. 12b an isotropic Maxwellian source $\left(T_{\perp}=T_{\|}\right)$is assumed, but the distribution in azimuth is taken to be nonuniform: $\Lambda=\Lambda_{2}$ with $m=2$. The fact that the source is isotropic causes the anisotropy to approach its critical value more rapidly and the growth rate peaks have shorter duration. This illustrates the fact that even for an isotropic population of injected plasma, an anisotropic distribution may be observed at later local times due to energy dispersion if the plasma is injected non-uniformly in azimuth.

The general shape of the curves in Fig. 12 may be understood with reference to Fig. 13: the initial rise is due to an increase in $\eta$, resulting from the presence of electrons with progressively lower energies and hence higher densities (closer to the peak in the source distribution). The onset of enhanced emissions at a given frequency is thus a consequence of the rapid increase in the number of resonant particles. This should be contrasted with the results of Collier and Hughes (2004) where the upper edge of the envelope resulted from the $f_{B} / 2$ cutoff. The subsequent decrease in $\gamma$ results from the last term in Eq. (11), which diminishes as the anisotropy approaches its critical value.

A substantial variation in peak growth rate, amounting to several orders of magnitude, exists over the range of frequencies plotted in Fig. 12 due to the large difference in the density of the more energetic particles responsible for the lower frequency emissions and the lower energy particles associated with the higher frequencies. 


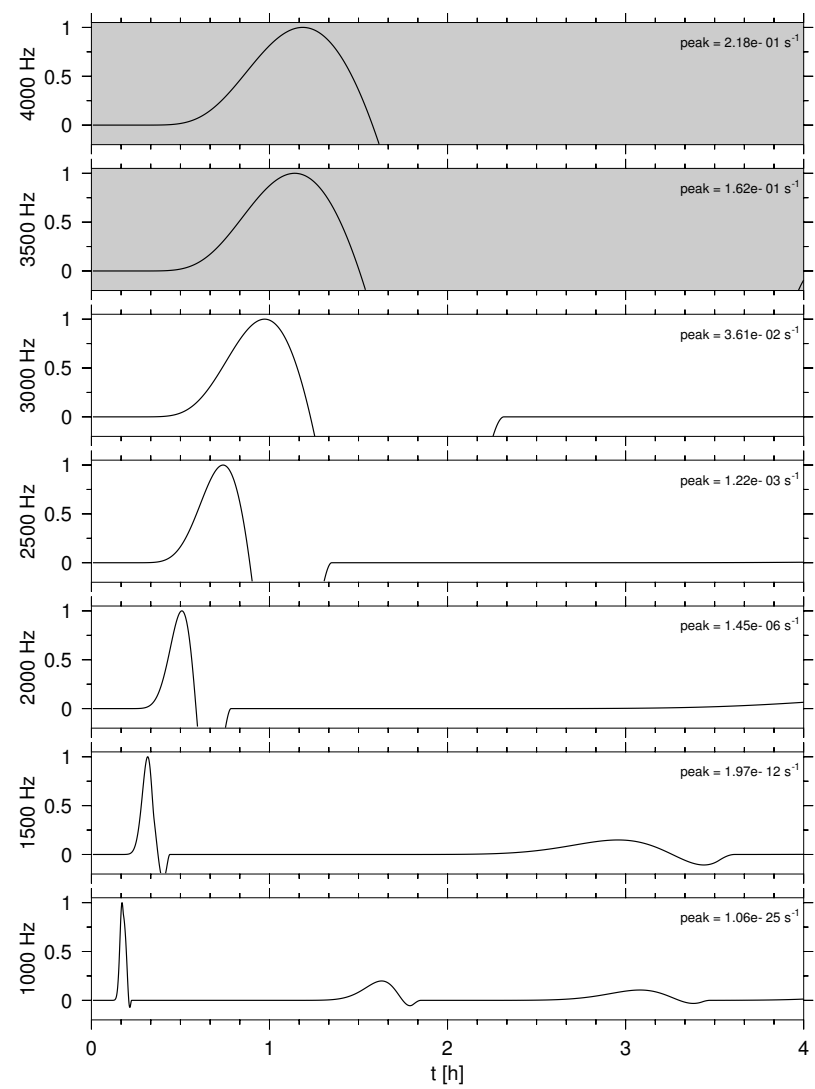

(a) bi-Maxwellian $\left(T_{\perp}=3 \mathrm{keV}, T_{\|}=2 \mathrm{keV}\right)$ and $\Lambda=\Lambda_{1}$

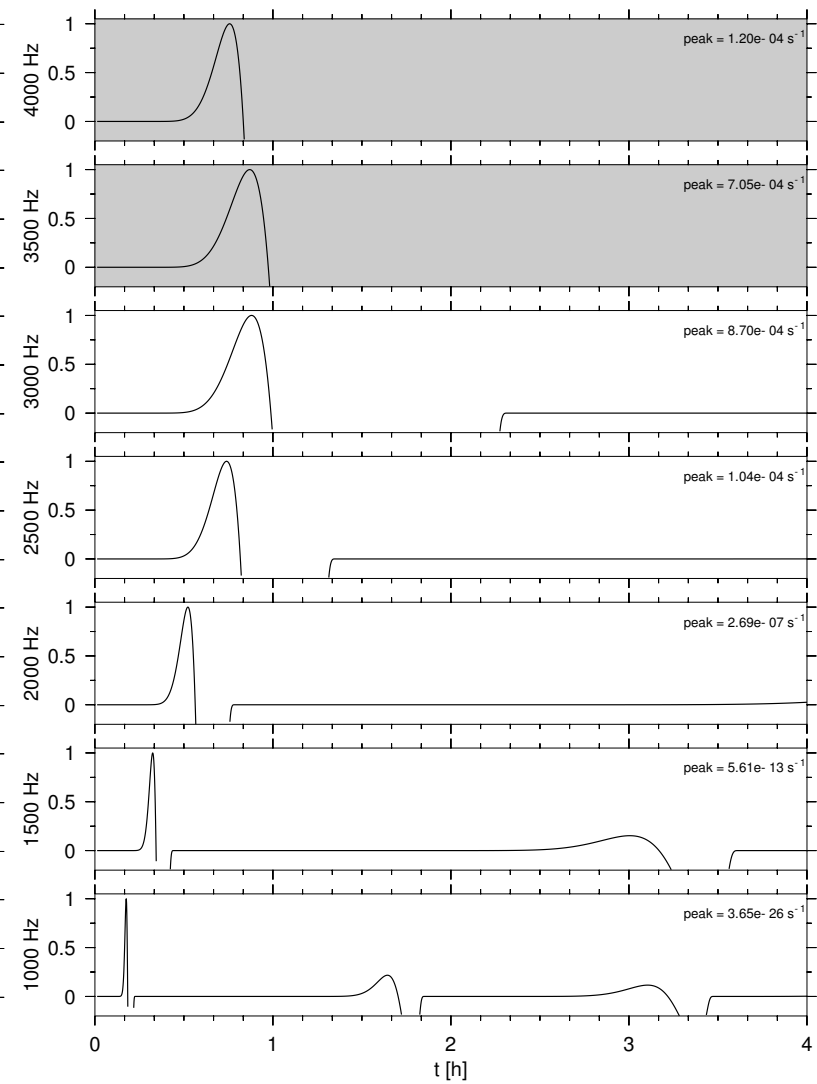

(b) isotropic Maxwellian $(T=2 \mathrm{keV})$ and $\Lambda=\Lambda_{2}$

Fig. 12. Normalised growth rate, $\gamma / \gamma_{\max }$ for an observer at $\psi_{0}=45^{\circ}$ and $L=5$. The upper two panels are shaded to indicate that these represent frequencies above $f_{B} / 2$, which are not observable on the ground. The plots indicate that at each frequency an interval of wave growth occurs; these intervals arise with greater delay at higher frequencies.

The range in $\gamma$ for the frequencies considered may be reduced by selecting higher temperatures for the source distribution. This, however, also results in the duration of the events declining dramatically.

One should note that following the growth rate peaks in Fig. 12, large negative excursions occur which are truncated by the axes of the plots. These arise as the distribution of particles at the observation point shifts to lower energies, the anisotropy approaches, then drops below the critical value, $A_{c}$, and the final term in Eq. (11) becomes negative. However, the lower energy particles are also drawn from closer to the peak in the injected particle distribution and consequently lead to large values of $\eta$ and hence $\gamma \ll 0$. Wave growth is only expected for $A>A_{c}$ : once the anisotropy drops below this critical value, amplification of VLF waves ceases and damping may occur.

The trend of peak delay times increasing with frequency is reversed at higher frequencies. The origin of this effect is as follows: initially, the anisotropy at all $W_{\|}$is large. As time progresses the anisotropies decrease, with those corresponding to resonance at higher frequencies declining least rapidly. However, the critical anisotropy, which starts to

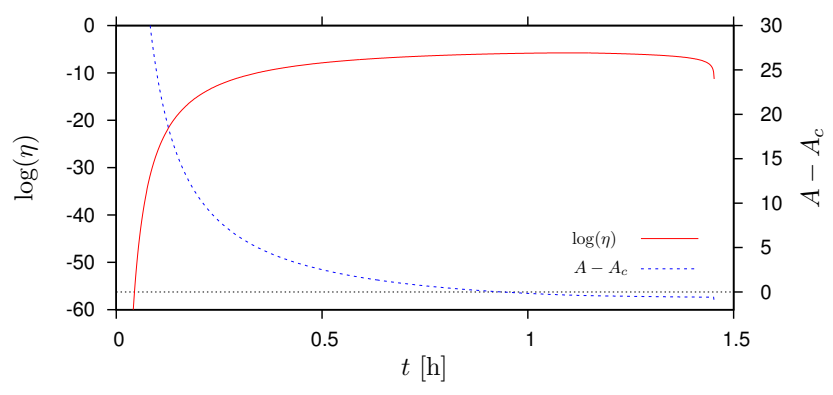

Fig. 13. Factors contributing to the shape of the growth rate curves for the source distribution applicable to Fig. $12 \mathrm{a}$ at $W_{\|}=20 \mathrm{keV}$. The initial rise in $\gamma$ is associated with the increase in $\eta$ as more particles with the specified $W_{\|}$drift into the station's field of view. The subsequent decrease in $\gamma$ occurs as the anisotropy of these particles approaches its critical value.

increase rapidly with frequency for $f / f_{B} \gtrsim 0.5$, is larger at higher frequencies and consequently, above a certain frequency, which depends on both $L$ and the particulars of the source, the difference $A-A_{c}$ is reduced more rapidly with time. 


\subsection{Lorentzian source}

Proceeding next to a Lorentzian source distribution with $T=2 \mathrm{keV}$ and $p=1$. The selection of larger or smaller $T$ effects a shift in the dominant emission frequency to lower or higher values respectively. Although the inherent anisotropy of this distribution, $A=1$, is large, it is not atypical of plasma in the magnetosphere (Hargreaves, 1992).

In Fig. 14 the normalised growth rate, $\gamma / \Omega$, is plotted as a function of frequency and time for a selection of $L$. These growth rate spectrograms are most reminiscent of the observed events, exhibiting a band of emissions with well-defined upper and lower cutoffs, and a progression towards higher frequencies. The duration of the period of wave amplification and the range of frequencies concerned are consistent with observations. Comparison of the growth rates calculated here with the peak value of $\gamma / \Omega=1.4 \times 10^{-2}$ determined from GEOS 2 data by Cornilleau-Wehrlin et al. (1985), reveals that this model produces relatively small values of $\gamma$, yet these scale directly with the choice of $n_{h}$.

Smith et al. (1999) observe that the amplitude of the signal begins to rise above the ambient level simultaneously in all VELOX channels, with the rate of increase diminishing at higher frequencies. This could be consistent with wave amplification within the injection region (Smith et al., 1999) but may also be accounted for by the rapid drift of the most energetic particles. The latter leads to the simulated growth rate rising above zero immediately following injection at all frequencies for which $A>A_{c}$.

A far greater uniformity in peak growth rates is evident across the range of frequencies in Fig. 14 as compared with the results for Maxwellian injections. This originates from the augmented high energy tail in the Lorentzian distribution which admits more electrons in resonance with low frequency waves. Source distributions with smaller $\kappa$ produce more substantial wave growth at lower frequencies, while as $\kappa \rightarrow \infty$ the Lorentzian distribution approaches a Maxwellian and the amplification of lower frequencies is curtailed.

In Fig. 14 it is apparent that a frequency exists above which there is no wave amplification. This frequency corresponds to that $W_{\|}$for which the anisotropy is always sub-critical. The fact that this effective upper cutoff frequency falls at approximately half the gyrofrequency is entirely coincidental. Modification of the degree of anisotropy in the source distribution leads to variation in this cutoff frequency and in the duration of the emissions. In particular, increasing $p$ results in electrons with large pitch angles forming a more substantial proportion of the population, and consequently, a larger range of frequencies exists for which the anisotropy is above the critical threshold and a more persistent event occurs because $A>A_{c}$ for a longer period.

Since the azimuthal drift rate is proportional to $L$, it is moderately surprising that the duration of the events appears to increase with $L$. This may be understood as follows: for a fixed frequency the resonant energy decreases more rapidly than $L^{-1}$, and hence the drift rate, proportional to both $W$ and $L$, of particles resonant with a particular frequency wave

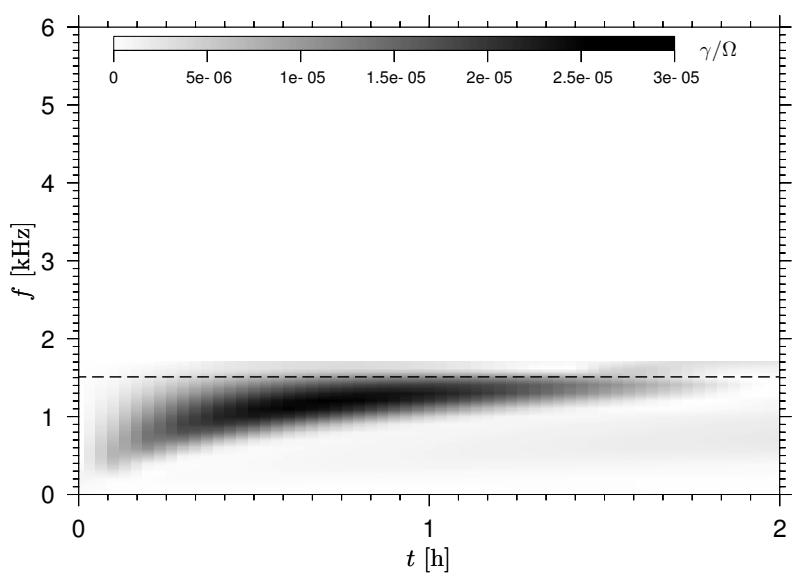

(a) $L=6.6$

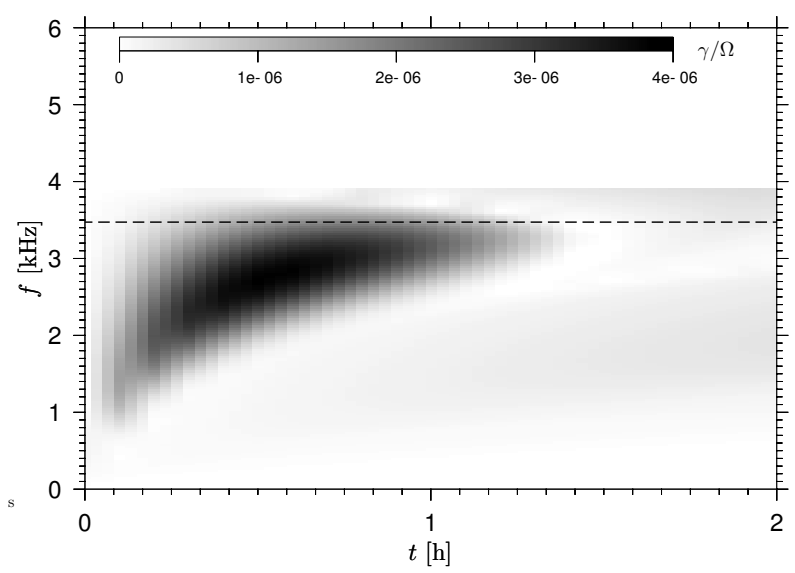

(b) $L=5.0$

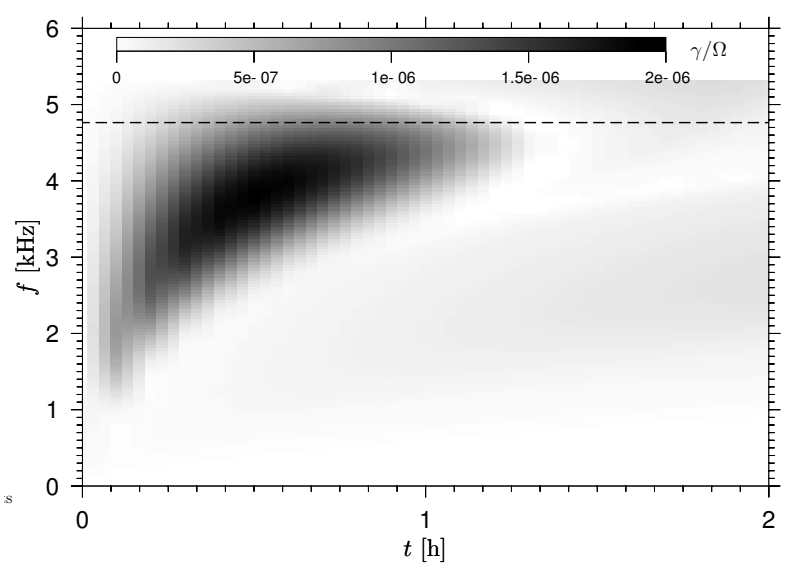

(c) $L=4.5$

Fig. 14. Normalised growth rate, $\gamma / \Omega$, for a Lorentzian source distribution function with $T=2 \mathrm{keV}, \kappa=2$ and $p=1$. Local time distribution of the injection is described by $\Lambda=\Lambda_{2}$. Observing station located at $\psi_{0}=45^{\circ}$ and source width $\delta \psi=45^{\circ}$. Dashed line indicates $f=f_{B} / 2$.

declines with increasing $L$. The modelled frequency sweep rate therefore varies inversely with $L$. The range of $d f / d t$ observed by Smith et al. (1996) could thus be accounted for by particles drifting on different $L$ shells. 


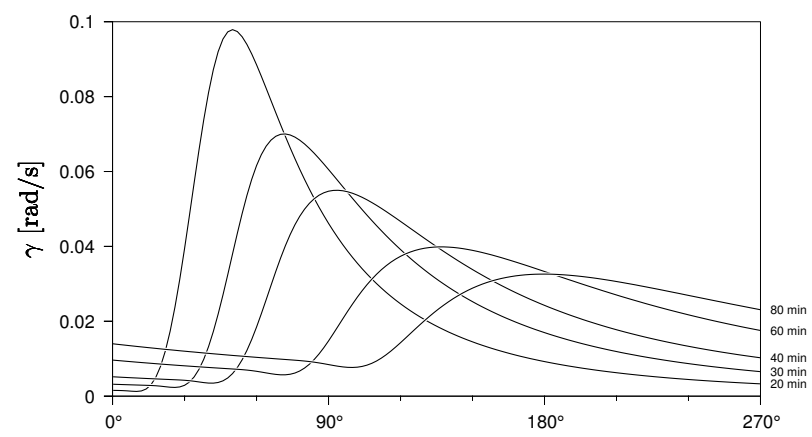

(a) growth rate

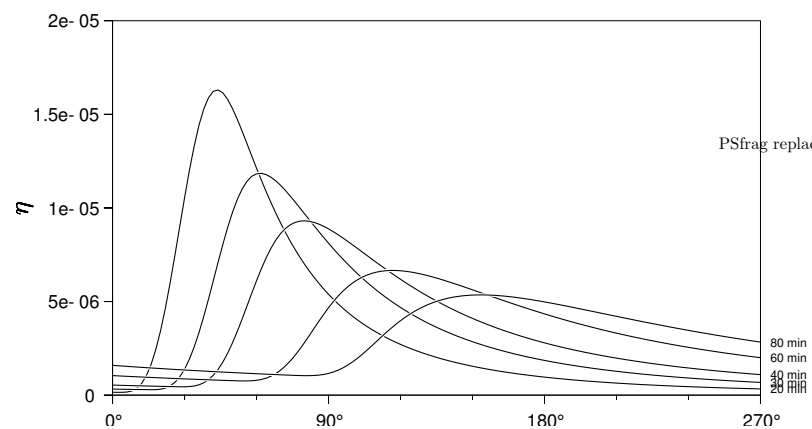

(b) number of particles in resonance

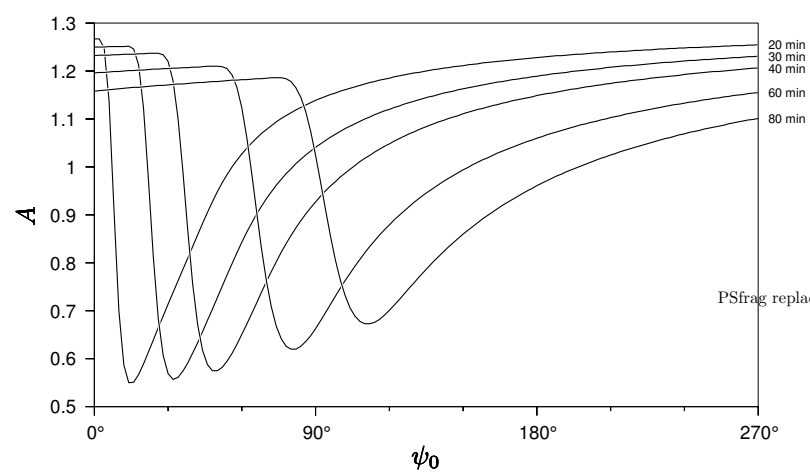

(c) anisotropy

Fig. 15. Variation of (a) growth rate, (b) $\eta$ and (c) anisotropy with the local time of the observer for electrons with $W_{\|}=40 \mathrm{keV}$ and $\delta \psi=45^{\circ}$ at $L=5$.

\subsection{Observer local time}

The variation in the anisotropy, $\eta$ and growth rate with the station local time is plotted in Fig. 15. At a fixed $\psi_{0}$ the anisotropy is initially maximised, it decreases with time until it reaches a minimum from which it recovers rather rapidly. The initial decrease is effected by electrons that have drifted directly from the source to the observer. However, as this population approaches cutoff, those electrons which have completed a full orbit around the Earth before returning to the observer begin to exert a larger influence. As time proceeds, the peaks in the curves for $\eta$ shift to later local times as particles with the specified $W_{\|}$drift eastwards. The peaks become progressively less sharp as these particles encompass a range of $\alpha$ (and hence $W$ ) and dispersive drift therefore spreads

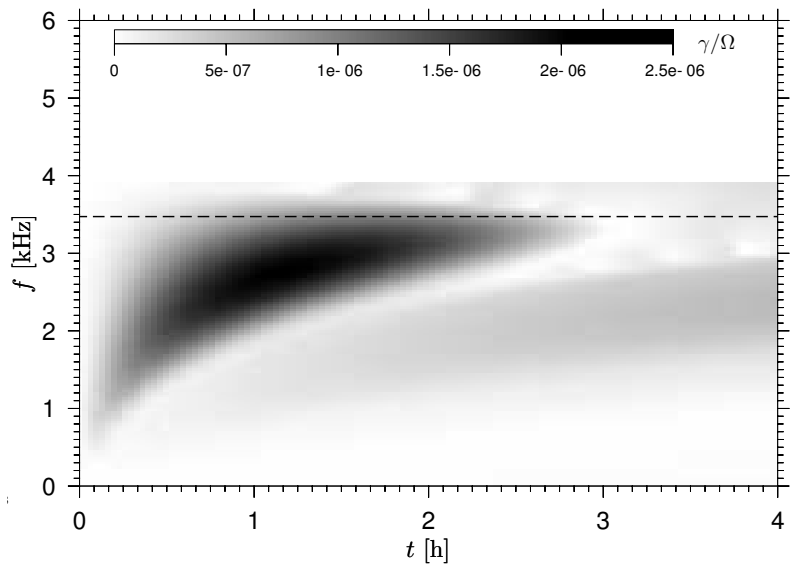

(a) dawn $\left(\psi_{0}=90^{\circ}\right)$

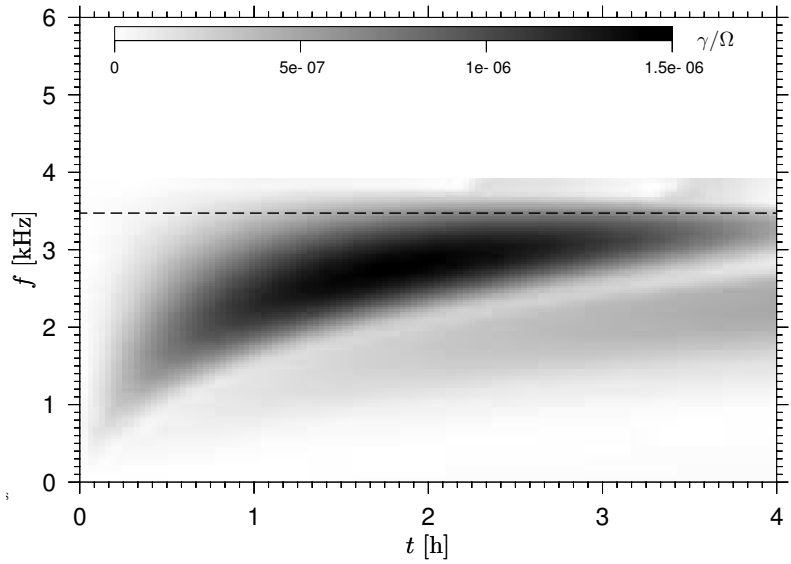

(b) morning sector $\left(\psi_{0}=135^{\circ}\right)$

Fig. 16. Normalised growth rate, $\gamma / \Omega$, for an observer (a) at dawn and (b) in the morning sector on $L=5$ for the source described in Fig. 14.

them out in local time. As noted by Jentsch (1976), the fact that the anisotropy increases with local time displaces the peaks in the growth rate towards later local times with respect to the peaks in $\eta$.

Figure 15a indicates that the largest growth rates are predicted for stations located at the earliest local times. This is confirmed by a comparison of Figs. 14b, 16a and b, which are each separated by 3-h of local time, and illustrate a systematic reduction in peak growth rate. The reduced growth rate is brought about by the dispersion of the particles, with the population becoming spread out in local time and fewer particles being present at a given instant for an observer located further east of midnight.

The broader growth rate peaks predicted at later local times imply that events of longer duration should be expected for larger $\psi_{0}$. This effect is also demonstrated in Figs. 14b, $16 a$ and $b$. A natural consequence of a model based on energy dispersion is that for observers located at later local times, the peaks at higher frequencies should occur with greater delays, suggesting that SCE with lower slopes should be anticipated at stations located further from midnight. Although 
this effect, noted by Hughes (1995), is not clearly evident in the more extensive data of Smith et al. (1996, Fig. 11), it is indeed produced in the current model. Failure to clearly discern this trend experimentally may be attributable to variability in the factors characterising individual substorms.

The extent of the injection region does not prove to be a particularly significant factor in determining the duration of the modelled SCE, although a small extension of the emission period is apparent for injections distributed over a larger range of local times. The event duration is, however, sensitive to the local time of the observer, with longer events observed at later local times.

\section{Conclusion}

A simple model has been presented which examines the variation of the electron population on an observer's meridian resulting from the gradient-curvature and corotation drift of plasma injected around midnight. No dawn-dusk electric field was considered nor were the effects of the atmospheric loss cone or other loss mechanisms taken into account. Subject to these conditions the anisotropy and whistler mode growth rate resulting from the cyclotron resonance interaction were calculated using the formulation of Kennel and Petschek (1966).

As noted by Cornilleau-Wehrlin et al. (1985), an analysis of this sort accounts for waves of a continuous broadband nature but does not describe the detailed frequency-time structure of chorus emissions, which consist of numerous short duration rising or falling tones. However, examination of the data presented in Fig. 1 over much shorter time scales reveals that the SCE may be composed of either discrete elements, consistent with the denomination of this event type, or broad-band emissions with very little structure. The model considered here is most applicable in the latter case.

Collier and Hughes (2004) described SCE simulations based on a particle tracking approach, tracing the motion of injected electrons subject to $\boldsymbol{E} \times \boldsymbol{B}$ and gradient-curvature drifts. The effect of the radial motion of the injected population resulting from a dawn-dusk convection electric field was investigated. The conclusions of Smith et al. (1996) and Abel et al. (2002) regarding the limited contribution of dispersive drift to the SCE mechanism were supported by the results of Collier and Hughes (2004), where it was determined that a relatively large convection electric field was required to produce results resembling the observations. However, Collier and Hughes (2004) treated the action of each electron individually and a realistic assessment of the anisotropy and growth rate, which depend on the whole ensemble of particles, was not performed.

The model considered here neglects the convection electric field and treats only the azimuthal motion of the electrons. Furthermore, whereas Collier and Hughes (2004) only crudely accounted for the instability conditions, the present calculations accurately determine the growth rate arising from the anisotropy of the relevant portion of the electron population. The results presented here, in which a qualitative similarity between the modelled growth rate spectrograms and VLF observations is immediately apparent, suggest that it is possible to produce emissions with the characteristics of a SCE in the absence of a convection electric field, indicating that dispersive drift may indeed contribute substantially to the SCE mechanism.

A process has emerged which accounts for the rising frequency of the lower boundary of the SCE envelope. This transpires from a cutoff in the maximum $W_{\|}$for electrons at the observer which decreases with time, and a consequent increase in the minimum possible resonance frequency.

The quoted results suggest certain characteristics of an injected electron population which would favour the generation of a SCE. If the injected particles are distributed uniformly over a segment of local time, then the population must be inherently anisotropic. A source distribution with a significant high energy tail produces more uniform growth rates over a range of frequencies. However, in the case of such a non-thermal distribution, it appears necessary to assume that the injected population is anisotropic in order for the dispersive drift to achieve an anisotropy greater than the critical threshold for a suitable frequency spectrum. In view of the fact that the spectrum of injected particles exhibits both upper and lower cutoffs, neither the Maxwellian nor Lorentzian description is entirely appropriate and further investigation should focus on a better characterisation of the injected population.

Acknowledgements. We gratefully acknowledge G. D. Reeves and M. F. Thomsen of LANL for providing the geosynchronous particle data. We are indebted to A. J. Smith and M. P. Freeman of the British Antarctic Survey for supplying Halley VELOX and magnetometer data. We thank the Sodankylä Geophysical Observatory for the provision of riometer data.

Topical Editor T. Pulkkinen thanks G. Abel and F. Lefeuvre for their help in evaluating this paper.

\section{References}

Abel, G. A., Smith, A. J., Meredith, N. P., and Anderson, R. R.: The Evolution of Substorm-enhanced whistler mode Waves and Their Relation to SCEs Seen on the Ground, in Proceedings of the Sixth International Conference on Substorms, University of Washington, Seattle, 2002.

Akasofu, S.-I.: Dynamics of the Magnetosphere, D. Reidel Publishing Company, 1980.

Birn, J., Thomsen, M. F., Borovsky, J. E., Reeves, G. D., McComas, D. J., and Belian, R. D.: Characteristic plasma properties during dispersionless substorm injections at geosynchronous orbit, J. Geophys. Res., 102, 2309-2324, 1997.

Birn, J., Thomsen, M. F., Borovsky, J. E., Reeves, G. D., McComas, D. J., Belian, R. D., and Hesse, M.: Substorm electron injections: Geosynchronous observations and test particle simulations, J. Geophys. Res., 103, 9235-9248, 1998.

Bracewell, R. N.: The Fourier Transform and its Applications, McGraw-Hill Book Company, 1965.

Carpenter, D. L.: Whistler Studies of the Plasmapause in the Magnetosphere, 1. Temporal Variations in the Position of the Knee 
and Some Evidence on Plasma Motions near the Knee, J. Geophys. Res., 71, 693-709, 1966.

Carpenter, D. L. and Anderson, R. R.: An ISEE/Whistler Model of Equatorial Electron Density in the Magnetosphere, J. Geophys. Res., 97, 1097-1108, 1992.

Carpenter, D. L., Fraser-Smith, A. C., Unwin, R. S., Hones, Jr, E. W., and Heacock, R. R.: Correlation between Convection Electric Fields in the Nightside Magnetosphere and Several Wave and Particle Phenomena during Two Isolated Substorms, J. Geophys. Res., 76, 7778-7786, 1971.

Carpenter, D. L., Foster, J. C., Rosenberg, T. J., and Lanzerotti, L. J.: A Subauroral and Mid-Latitude View of Substorm Activity, J. Geophys. Res., 80, 4279-4286, 1975.

Collier, A. B. and Hughes, A. R. W.: Digital VLF Recording and Analysis System for SANAE-IV, South African Journal of Science, 98, 547-550, 2002.

Collier, A. B. and Hughes, A. R. W.: Modelling and Analysis of Substorm-Related Chorus Events, Advances in Space Research, 34, 1819-1823, 2004.

Cornilleau-Wehrlin, N., Solomon, J., Korth, A., and Kremser, G.: Experimental Study of the Relationship between Energetic Electrons and ELF Waves Observed on Board GEOS: A Support to Quasi-Linear Theory, J. Geophys. Res., 90, 4141-4154, 1985.

Ejiri, M.: Trajectory Traces of Charged Particles in the Magnetosphere, J. Geophys. Res., 83, 4798-4810, 1978.

Etcheto, J., Gendrin, R., Solomon, J., and Roux, A.: A SelfConsistent Theory of Magnetospheric ELF Hiss, J. Geophys. Res., 78, 8150-8166, 1973.

Foster, J. C. and Rosenberg, T. J.: Electron Precipitation and VLF Emissions Associated With Cyclotron Resonance Interactions Near the Plasmapause, J. Geophys. Res., 81, 2183-2192, 1976.

Friedel, R. H. W., Korth, A., and Kremser, G.: Substorm onsets observed by CRRES: Determination of energetic particle source regions, J. Geophys. Res., 101, 13 137-13 154, 1996.

Hargreaves, J. K.: The solar-terrestrial environment, Cambridge Atmospheric and Space Science Series, Cambridge University Press, 1992.

Hayakawa, M., Yamanaka, Y., Parrot, M., and Lefeuvre, F.: The Wave Normals of Magnetospheric Chorus Emissions Observed on Board GEOS 2, J. Geophys. Res., 89, 2811-2821, 1984.

Helliwell, R. A.: Whistlers and Related Ionospheric Phenomena, Stanford University Press, 1965.

Henderson, M. G., Reeves, G. D., Spence, H. E., Sheldon, R. B., Jorgensen, A. M., Blake, J. B., and Fennell, J. F.: First energetic neutral atom images from Polar, Geophys. Res. Lett., 24, 11671170, 1997.

Horne, R. B. and Thorne, R. M.: Relativistic electron acceleration and precipitation during resonant interactions with whistler mode chorus, Geophys. Res. Lett., 30, 2003.

Hughes, A. R. W.: VLF Waves in the Magnetosphere, Astrophys. Space Sci., 230, 431-438, 1995.

Inan, U. S., Platino, M., Bell, T. F., Gurnett, D. A., and Pickett, J. S.: Cluster measurements of rapidly moving sources of ELF/VLF chorus, J. Geophys. Res., 109, 2004.

Isenberg, P. A., Koons, H. C., and Fennell, J. F.: Simultaneous Observations of Energetic Electrons and Dawnside Chorus in Geosynchronous Orbit, J. Geophys. Res., 87, 1495-1503, 1982.

Jentsch, V.: Electron Precipitation in the Morning Sector of the Auroral Zone, J. Geophys. Res., 81, 135-146, 1976.

Kennel, C. F. and Petschek, H. E.: Limit on Stably Trapped Particle Fluxes, J. Geophys. Res., 71, 1-28, 1966.

Kivelson, M. G.: Magnetospheric Electric Fields and Their Varia- tion with Geomagnetic Activity, Rev. Geophys. Space Phys., 14, 189-197, 1976.

Li, X., Baker, D. N., Temerin, M., Reeves, G. D., and Belian, R. D.: Simulation of dispersionless injections and drift echoes of energetic electrons associated with substorms, Geophys. Res. Lett., 25, 3763, 1998.

Li, X., Sarris, T. E., Baker, D. N., Peterson, W. K., and Singer, H. J.: Simulation of energetic particle injections associated with a substorm on 27 August, 2001, Geophys. Res. Lett., 30, 2003.

Liemohn, H. B.: Cyclotron-Resonance Amplification of VLF and ULF Whistlers, J. Geophys. Res., 72, 39-55, 1967.

Maeda, K. and Lin, C. S.: Frequency Band Broadening of Magnetospheric VLF Emissions Near the Equator, J. Geophys. Res., 86, 3635-3639, 1981.

Mauk, B. H. and McIlwain, C. E.: Correlation of $K_{p}$ With the Substorm-Injected Plasma Boundary, J. Geophys. Res., 79, 3193-3196, 1974.

McCormac, B. M.: Magnetospheric Physics, D. Reidel Publishing Company, 1974.

McIlwain, C. E.: Coordinates for Mapping the Distribution of Magnetically Trapped Particles, J. Geophys. Res., 66, 3681-3691, 1961.

McIlwain, C. E.: Substorm Injection Boundaries, in McCormac (1974), 143-154, 1974.

Meredith, N. P., Horne, R. B., and Anderson, R. R.: Substorm dependence of chorus amplitudes: Implications for the acceleration of electrons to relativistic energies, J. Geophys. Res., 106, $13165,2001$.

Nunn, D., Omura, Y., Matsumoto, H., Nagano, I., and Yagitani, S.: The numerical simulation of VLF chorus and discrete emissions observed on the Geotail satellite using a Vlasov code, J. Geophys. Res., 102, 27 083-27 097, 1997.

Park, C. G., Lin, C. S., and Parks, G. K.: A Ground-Satellite Study of Wave-Particle Correlations, J. Geophys. Res., 86, 3753, 1981.

Parks, G. K., Mauk, B., Gurgiolo, C., and Lin, C. S.: Observations of Plasma Injection, in Akasofu (1980), 371-383, 1980.

Parrot, M., Santolík, O., Cornilleau-Wehrlin, N., Maksimovic, M., and Harvey, C. C.: Source location of chorus emissions observed by Cluster, Ann. Geophys., 21, 473-480, 2003.

Reeves, G. D.: New perspectives on substorm injections, in Proceedings of the Fourth International Conference on Substorms, edited by S. Kokubun and Y. Kamide, 785, Kluwer Academic Publishers, Hamanako, Japan, 1998.

Reeves, G. D., Belian, R. D., and Fritz, T. A.: Numerical Tracing of Energetic Particle Drifts in a Model Magnetosphere, J. Geophys. Res., 96, 13 997-14 008, 1991.

Reeves, G. D., Kettmann, G., Fritz, T. A., and Belian, R. D.: Further Investigation of the CDAW 7 Substorm Using Geosynchronous Particle Data: Multiple Injections and Their Implications, J. Geophys. Res., 97, 6417-6428, 1992.

Roederer, J. G.: Dynamics of Geomagnetically Trapped Radiation, Springer-Verlag, 1970.

Rosenberg, T. J., Siren, J. C., Matthews, D. L., Marthinsen, K., Holtet, J. A., Egeland, A., Carpenter, D. L., and Helliwell, R. A.: Conjugacy of Electron Microbursts and VLF Chorus, J. Geophys. Res., 86, 5819-5832, 1981.

Rycroft, M. J.: VLF emissions in the magnetosphere, Radio Sci., 7, 811-830, 1972.

Sazhin, S. S. and Hayakawa, M.: Magnetospheric Chorus Emissions: A Review, Planet. Space Sci., 40, 681-697, 1992.

Smirnova, N. A.: Fine Structure of the Ground-Observed VLF Cho- 
rus as an Indicator of the Wave-Particle Interaction Processes in the Magnetospheric Plasma, Planet. Space Sci., 32, 425-438, 1984.

Smith, A. J.: VELOX: a new VLF/ELF receiver in Antarctica for the Global Geospace Science mission, J. Atmos. Terr. Phys., 57, 507-524, 1995.

Smith, A. J., Freeman, M. P., and Reeves, G. D.: Postmidnight VLF chorus events, a substorm signature observed at the ground near $L=4$, J. Geophys. Res., 101, 24 641-24 653, 1996.

Smith, A. J., Freeman, M. P., Wickett, M. G., and Cox, B. D.: On the relationship between the magnetic and VLF signatures of the substorm expansion phase, J. Geophys. Res., 104, $12351-$ $12360,1999$.

Smith, A. J., Freeman, M. P., Hunter, S., and Milling, D. K.: VLF, magnetic bay, and Pi2 substorm signatures at auroral and midlatitude ground stations, J. Geophys. Res., 107, 1439, 2002.
Southwood, D. J. and Kivelson, M. G.: An Approximate Analytic Description of Plasma Bulk Parameters and Pitch Angle Anisotropy Under Adiabatic Flow in a Dipolar Magnetospheric Field, J. Geophys. Res., 80, 2069-2073, 1975.

Thomsen, M. F., Birn, J., Borovsky, J. E., Morzinski, K., McComas, D. J., and Reeves, G. D.: Two-satellite observations of substorm injections at geosynchronous orbit, J. Geophys. Res., 106, 84058416, 2001.

Tsurutani, B. T. and Smith, E. J.: Postmidnight Chorus: A Substorm Phenomenon, J. Geophys. Res., 79, 118-127, 1974.

Vasyliūnas, V. M.: A Survey of Low-Energy Electrons in the Evening Sector of the Magnetosphere with OGO 1 and OGO 3 , J. Geophys. Res., 73, 2839-2884, 1968. 